\title{
Fixed Parameter Algorithms for Planar Dominating Set and Related Problems*
}

\author{
Jochen Alber ${ }^{\dagger}$ Hans L. Bodlaender ${ }^{\ddagger}$ Henning Fernau ${ }^{\dagger} \quad$ Rolf Niedermeier $^{\dagger}$
}

\begin{abstract}
We present an algorithm that constructively produces a solution to the $k$-DOMINATING SET problem for planar graphs in time $O\left(c^{\sqrt{k}} n\right)$, where $c=3^{6 \sqrt{34}}$. To obtain this result, we show that the treewidth of a planar graph with domination number $\gamma(G)$ is $O(\sqrt{\gamma(G)})$, and that such a tree decomposition can be found in $O(\sqrt{\gamma(G)} n)$ time. The same technique can be used to show that the $k$-FACE COVER problem (find a size $k$ set of faces that cover all vertices of a given plane graph) can be solved in $O\left(c_{1}^{\sqrt{k}} n+n^{2}\right)$ time, where $c_{1}=2^{36 \sqrt{34}}$ and $k$ is the size of the face cover set. Similar results can be obtained in the planar case for some variants of $k$-DOMINATING SET, e.g., $k$-INDEPENDENT DOMINATING SET and $k$-WEIGHTED DOMINATING SET.
\end{abstract}

Keywords. NP-complete problems, fixed parameter tractability, planar graphs, PLANAR DOMINATING SET, FACE COVER, outerplanarity, treewidth.

*An extended abstract of this paper appeared in the proceedings of the 7th Scandinavian Workshop on Algorithm Theory (SWAT 2000), Springer-Verlag, LNCS 1851, pages 97-110, held in Bergen, Norway, July 5-7, 2000.

${ }_{\dagger}^{\dagger}$ Universität Tübingen, Wilhelm-Schickard-Institut für Informatik, Sand 13, D-72076 Tübingen, Federal Republic of Germany;

email: \{alber,fernau, niedermr\}@informatik.uni-tuebingen.de

Work of Jochen Alber supported by the DFG-research project "PEAL" (Parameterized complexity and Exact ALgorithms), NI 369/1-1; also, author to whom correspondence should be sent.

${ }^{\ddagger}$ Utrecht University, Department of Computer Science, Padualaan 14, De Uithof, 3584 CH Utrecht, The Netherlands; email:hansb@cs.uu.nl 


\section{Introduction}

A $k$-dominating set $D$ of an undirected graph $G$ is a set of $k$ vertices of $G$ such that each of the rest of the vertices has at least one neighbor in $D$. The minimum $k$ such that the graph $G$ has a $k$-dominating set is called the domination number of $G$, denoted by $\gamma(G)$. The $k$-DOMInATING SET problem, i.e., the task to decide, given a graph $G=(V, E)$ and a positive integer $k$, whether or not there exists a $k$-dominating set, is among the core problems in algorithms, combinatorial optimization, and computational complexity [4, 16, 27, 32, 44]. The problem is NP-complete, even when restricted to planar graphs with maximum vertex degree 3 and to planar graphs that are regular of degree 4 [27].

Coping with NP-hard problems. Despite their intractability, many NPhard problems are of great practical importance. Besides heuristic methods [39] which often lack theoretical analysis, the main contribution of theoretical computer science on the attack of intractability so far has been to design and analyze approximation algorithms $[4,32]$. The approximability of the DOMINATING SET problem has received considerable attention $[4,16,32]$. It is not known and it is not believed that DOMINATING SET for general graphs has a constant factor approximation algorithm (see, e.g., $[4,16]$ for details). The PLANAR DOMINATING SET problem (i.e., the dominating set problem restricted to planar graphs), however, possesses a polynomial time approximation scheme [5]. That is, there is a polynomial time approximation algorithm with approximation factor $1+\epsilon$, where $\epsilon$ is a constant arbitrarily close to 0 . However, the degree of the polynomial grows with $1 / \epsilon$. Hence, applying the approximation scheme does not necessarily lead to practical solutions. As an alternative, finding an "efficient" exact solution in "reasonable exponential time" for PLANAR DOMINATING SET is an interesting and promising research question.

Fixed parameter tractability. Lately, it has become popular to cope with computational intractability in a different way: parameterized complexity $[1,22$, $23,24]$. Here, the basic observation is that, for many hard problems, the seemingly inherent combinatorial explosion can be restricted to a "small part" of the input, the parameter. For instance, the $k$-VERTEX COVER problem can be solved by an algorithm with running time $O\left(k n+1.3^{k}\right)[14,41]$, where the parameter $k$ is a bound on the maximum size of the vertex cover set we are looking for and $n$ is the number of vertices in the given graph. The fundamental assumption is $k \ll n$. As can easily be seen, this yields an efficient, practical algorithm for small values of $k$. In general, a problem is called fixed parameter tractable if it can be solved in time $f(k) n^{O(1)}$ for an arbitrary function $f$ which depends only on $k$. Unfortunately, according to the theory of parameterized complexity, it is very unlikely that the $k$-DOMINATING SET problem is fixed parameter tractable. On the contrary, it was 
proven to be complete for $W[2]$ (see [20]), a "complexity class of parameterized intractability" (refer to Downey and Fellows [22] for details). However, PLANAR $k$-DOMINATING SET is fixed parameter tractable. This already easily follows from Baker's approximation result for PLANAR DOMINATING SET [5]. More precisely, Baker's method implies an $O\left(8^{3 k} n\right)$ time algorithm, where $n$ is the number of vertices. To obtain this result, note that a planar graph with a dominating set of size $k$ is at most $3 k$-outerplanar (see Section 2). Besides that, an $O\left(3^{9 k} n\right)$ time algorithm can be derived from this observation and the fact that an $r$ outerplanar graph has treewidth of at most $3 r-1$ (as exhibited in Section 2). Alternatively, the general logical framework of Frick and Grohe [26] easily proves the fixed parameter tractability of PLANAR $k$-DOMINATING SET. Downey and Fellows $[21,22]$ give an $O\left(11^{k} n\right)$ time algorithm, the so far best known time bound for PLANAR $k$-DOMINATING SET.

Relevance of (PLANAR) DOMINATING SET. The literature on DOMINATING SET problems in mathematics, computer science, and their applications (e.g., computational biology) is huge. Hence, we only refer to some surveys here, for instance, $[29,30,31,37,46]$. In particular, note that many papers have been published on domination problems for special graph classes [11] and/or variations of the fundamental problem, see, for example, $[2,6,12,13,15,25,17,34,35,40$, 49].

Our main result. We present fixed parameter tractability results for PLANAR $k$-DOMINATING SET and related problems. Our main result is to prove a new and perhaps surprising structural relationship: We show that a planar graph with a dominating set of size $k$ has treewidth $O(\sqrt{k})$. Up to now, only treewidth $O(k)$ was known, which is basically straightforward. Note that our proof can be made constructive. From this result, we then conclude that PLANAR $k$-DOMINATING SET can be solved in time $O\left(c^{\sqrt{k}} n\right)$, where $c=3^{6 \sqrt{34}}$. Clearly, the proven constant $c$ is huge and exhibits the limited usefulness of our result in practice. It is conceivable that this constant might be improved significantly due to refined analysis and/or additional algorithmic ideas. Also note that our analysis and, thus, the constant refers to a pure worst case scenario; it might say little about the average case performance or the behavior of our method on problem instances drawn from practical applications. Finally, and maybe most importantly, it should be emphasized that our result means a significant structural breakthrough. The best known running time for $k$-PLANAR DOMINATING SET was $O\left(c_{1}^{k} n\right)$ [21, 22], which we improved to $O\left(c^{\sqrt{k}} n\right)$. Indeed, this seems to be the first non-trivial result for an NP-hard, fixed parameter tractable problem at all where the exponent of the exponential term is growing sublinearly. 
Further contributions of our work. Our new method can also be used to significantly improve a known bound for the $k$-FACE COVER problem $[7,22,45]$. The problem is defined as follows $[22,7,45]$ : Given a plane graph $G$, i.e., a graph with a fixed embedding in the plane and a positive integer $k$, is there a set of at most $k$ faces (also called disks $[7,45]$ ), such that all of the graph vertices are covered by the faces, i.e., each vertex is part of at least one of these $k$ faces? The problem is NP-complete [7]. Downey and Fellows [22] gave an $O\left(12^{k} n\right)$ algorithm for this problem. For a slightly more general version of the problem, Bienstock and Monma [7] showed that there is a time $O\left(c_{2}^{k} n\right)$ algorithm, where $c_{2}$ is an unspecified constant. In this paper, we give an algorithm that solves $k$-FACE COVER in time $O\left(c_{3}^{\sqrt{k}} n+n^{2}\right)$, where $c_{3}=2^{36 \sqrt{34}}$. Finally, it is easy to extend our results for PLANAR DOMINATING SET to "PLANAR DOMINATING SET WITH PROPERTY $P$ " problems. For example, this includes the problems $k$ INDEPENDENT DOMINATING SET or $k$-TOTAL DOMINATING SET. In addition, our results can also be generalized to the weighted case with positive integer weights, i.e, PLANAR $k$-WEIGHTED DOMINATING SET.

Outline of the paper. Our paper is structured as follows. In Section 2, we introduce some key concepts for our work, including $r$-outerplanarity, layer decompositions of $r$-outerplanar graphs, and treewidth. Moreover, we point out the relations between domination number and $r$-outerplanarity, between $r$-outerplanarity and treewidth, and how to compute a minimum size dominating set given a tree decomposition of a graph. In Section 3, our main result follows, showing that for planar graphs with domination number $k$, a tree decomposition of width $O(\sqrt{k})$ exists. In addition, we also show how to actually construct such a tree decomposition. In Section 4, we summarize our findings in describing an algorithm solving the PLANAR DOMINATING SET problem in time $3^{O(\sqrt{k})} n$. In Section 5 , we extend our results to variations of PLANAR $k$-DOMINATING SET, PLANAR $k$ WEIGHTED DOMINATING SET, and, in particular, to the $k$-FACE COVER problem. We conclude the paper with some open problems and final remarks (Section 6).

\section{Preliminaries: Domination, $r$-outerplanarity, and treewidth}

In this section, we provide necessary notions and some known results. We assume familiarity with basic graph-theoretical notation as provided in $[19,38]$. In particular, for a graph $G=(V, E)$ and a subset $V^{\prime} \subseteq V$, the subgraph induced by the vertices $V \backslash V^{\prime}$ will frequently be denoted by $G-V^{\prime}$. If $V^{\prime}=\{v\}$ is a singleton, we write $G-v$ instead of $G-\{v\}$.

This section is split into three parts. The first subsection introduces the notion of $r$-outerplanarity and the concept of a layer-decomposition of a planar graph. 
Moreover, we state a central observation relating the domination number and the outerplanarity number of a planar graph. In the second subsection, we provide the concepts of tree decompositions and treewidth. Also, we explicitly outline how the Dominating Set problem can be solved in this framework. This idea will be used as a main tool for our algorithm. In the third subsection, we outline how to construct a tree decomposition of small width for $r$-outerplanar graphs, which is used as an important lemma in later parts.

\subsection{Domination and $r$-outerplanarity}

Definition 1 A crossing-free embedding of a graph $G$ in the plane is called outerplanar if each vertex lies on the boundary of the outer face. A graph $G$ is called outerplanar if it admits an outerplanar embedding in the plane.

The following generalization of the notion of outerplanarity was introduced by Baker [5].

Definition 2 A crossing-free embedding of a graph $G$ in the plane is called $r$-outerplanar if, for $r=1$, the embedding is outerplanar, and, for $r>1$, inductively, when removing all vertices on the boundary of the outer face and their incident edges, the embedding of the remaining subgraph is $(r-1)$-outerplanar. A graph $G$ is called $r$-outerplanar if it admits an $r$-outerplanar embedding. The smallest number $r$, such that $G$ is $r$-outerplanar is called the outerplanarity number.

In this way, we may speak of the layers $L_{1}, \ldots, L_{r}$ of an embedding of an $r$ outerplanar graph.

Definition 3 For a given $r$-outerplanar embedding of a graph $G=(V, E)$, we define the $i$ th layer $L_{i}$ inductively as follows. Layer $L_{1}$ consists of the vertices on the boundary of the outer face, and, for $i>1$, the layer $L_{i}$ is the set of vertices that lie on the boundary of the outer face in the embedding of the subgraph $G-\left(L_{1} \cup \ldots \cup L_{i-1}\right)$.

One easily observes the following central relation between the domination number and the outerplanarity number of a planar graph.

Proposition 4 If a planar graph $G=(V, E)$ has a $k$-dominating set, then all plane embeddings of $G$ can be at most $3 k$-outerplanar.

Proof. Note that, for a given crossing-free embedding of $G$ in the plane, each vertex in the dominating set can dominate vertices from the previous, the next, or its own layer only. Hence, each vertex in the dominating set can contribute to at most 3 new layers.

To understand the techniques used in the following sections, it is helpful to consider the concept of a layer decomposition of an $r$-outerplanar embedding of 
graph $G$. It is a forest of height $r-1$ which is defined as follows: the nodes of the trees are sets of vertices of $G$ and the different trees correspond to different components of $G$. In general, the $i$ th layer of the layer decomposition forest defines a set of vertices $L_{i}$, namely, the $i$ th layer of $G$.

Consider now the $i$ th layer of the forest, i.e., the nodes of level $i$ in the decomposition forest consisting of, possibly, several vertex sets $C_{i, 1}, \ldots, C_{i, \ell_{i}}$. In other words, $L_{i}=\bigcup_{j=1}^{\ell_{i}} C_{i, j}$. The vertex sets $C_{i, 1}, \ldots, C_{i, \ell_{i}}$ correspond to the vertices of different components of the subgraph induced by $L_{i}$. We refer to $C_{i, j}$ as a layer-component. In particular, the first layer consists of layer-components each of which equals the vertices from $L_{1}$ of one particular component.

We need some further notation for the construction in the next sections.

Definition 5 A layer-component $C_{i, j}$ of layer $L_{i}$ is called non-vacuous if there are vertices from layer $L_{i+1}$ in the interior of $C_{i, j}$ (i.e., in the region enclosed by the subgraph induced by $C_{i, j}$ ).

Lemma 6 Let $\emptyset \neq C \subseteq C_{i, j}$ be a subset of a non-vacuous layer-component $C_{i, j}$ of layer $i$, where $i \geq 2$. Then there exists a unique smallest (in number of vertices) cycle $B(C)$ in layer $L_{i-1}$, such that $C$ is contained in the region enclosed by $B(C)$. No other vertex of layer $L_{i-1}$ is contained in this region.

Proof. The existence of a cycle $B^{\prime}(C)$ in layer $L_{i-1}$ which encloses $C$ follows easily from the definition of a layer. Note, that the region enclosed by $B^{\prime}(C)$, again by the definition of a layer, cannot contain vertices of layer $L_{i-1}$. In order to obtain the smallest such cycle, we proceed as follows. Let $B^{\prime}(C)=\left(w_{1}, \ldots, w_{m}\right)$. If there exists a pair of vertices $w_{i}, w_{j}$ with $j>i$ and $|i-j| \notin\{1, m-1\}$ that happen to be neighbors in the graph, and if $C$ is enclosed by one of the cycles $\left(w_{i}, \ldots, w_{j}\right)$ or $\left(w_{j}, \ldots, w_{m}, w_{1}, \ldots, w_{i}\right)$, then we exchange $B^{\prime}(C)$ by this one. Continuing this procedure until no further such situation occurs it is not hard to verify that we end up with a smallest cycle that encloses $C$.

Definition 7 For each non-empty subset $C$ of a non-vacuous layer-component of layer $i(i \geq 2)$, the set $B(C)$ as given in Lemma 6 is called the boundary cycle of $C$.

\subsection{Domination and treewidth}

The main tool we use in our algorithm is the concept of tree decompositions as, e.g., described in [9].

Definition 8 Let $G=(V, E)$ be a graph. A tree decomposition of $G$ is a pair $\left\langle\left\{X_{i} \mid i \in I\right\}, T\right\rangle$, where each $X_{i}$ is a subset of $V$, called a $b a g$, and $T$ is a tree with the elements of $I$ as nodes. The following three properties should hold: 
1. $\bigcup_{i \in I} X_{i}=V$;

2. for every edge $\{u, v\} \in E$, there is an $i \in I$ such that $\{u, v\} \subseteq X_{i}$;

3. for all $i, j, k \in I$, if $j$ lies on the path between $i$ and $k$ in $T$, then $X_{i} \cap X_{k} \subseteq$ $X_{j}$.

The width of $\left\langle\left\{X_{i} \mid i \in I\right\}, T\right\rangle$ equals $\max \left\{\left|X_{i}\right| \mid i \in I\right\}-1$. The treewidth of $G$ is the minimum $k$ such that $G$ has a tree decomposition of width $k$.

In Section 2.3 we give a constructive proof of a result stated in [36, Table 2, page 550] and in [10, Theorem 83]:

Theorem 9 An r-outerplanar graph has treewidth of at most $3 r-1$.

Proposition 4 and Theorem 9 immediately imply the following relation between the domination number and the treewidth of a planar graph.

Corollary 10 If a planar graph $G=(V, E)$ has a $k$-dominating set, then it has bounded treewidth, or, more precisely, its treewidth is bounded by $9 k-1$.

We will give a considerably stronger bound later.

The concept of tree decompositions can be used to solve various graph problems. Typically, treewidth based algorithms proceed in two stages: The first stage finds a tree decomposition of bounded width of the input graph, and the second stage solves the problem using dynamic programming approaches on the tree decomposition (see [9]).

As to the first stage, the problem to determine whether a graph has treewidth bounded by some constant $\ell$ and, if so, producing a corresponding tree decomposition, has, at the current state of research, no algorithmically feasible solution. Even though the problem is proven to be fixed parameter tractable, the constants in the algorithm presented in [8] are too large for practical purposes. However, in the case of PLANAR $k$-DOMINATING SET, this first stage of the tree decomposition algorithm is less involved, as will be shown in Theorem 12 .

The second stage can be proceeded in polynomial time with a factor depending on the bound of the treewidth. The corresponding result for the DOMinating SET problem is the following. Note that, in this situation, we do not need to assume planarity of the underlying graph.

Theorem 11 If a tree decomposition of width $\ell$ of a graph is known, then a minimum dominating set can be determined in time $3^{\ell} N$, where $N$ is the number of nodes of the tree decomposition. 
Proof. The theorem can be proven by using dynamic programming techniques, as described in a more general context, e.g., in [9, 48]. For the sake of preciseness, we outline how these techniques apply to solving DOMINATING SET in the claimed running time.

Let $\mathcal{X}=\left\langle\left\{X_{i} \mid i \in I\right\}, T\right\rangle$ be a tree decomposition for the graph $G=(V, E)$. We assume that the vertices in the bags are given some order. For each bag $X_{i}=\left(x_{1}^{(i)}, \ldots, x_{n_{i}}^{(i)}\right)$ with $n_{i}:=\left|X_{i}\right|$, we keep a table $A_{i}$ of size $3^{n_{i}} \times\left(n_{i}+1\right)$. Each of the $3^{n_{i}}$ rows represents a coloring of the vertices in $X_{i}$ using the 3 distinct colors

- "black" (represented by 0, meaning that the vertex belongs to the dominating set),

- "grey" (represented by 1, meaning that the vertex is dominated but does not (yet) belong to the dominating set), and

- "white" (represented by 2, meaning that the vertex still needs to be dominated).

More precisely, for each $j \in\left\{1, \ldots, 3^{n_{i}}\right\}$, we define the coloring

$$
C_{j}^{(i)}:=\left(c_{j, 1}^{(i)}, \ldots, c_{j, n_{i}}^{(i)}\right) \in\{0,1,2\}^{n_{i}}, \quad \text { such that } j-1=\sum_{t=1}^{n_{i}} c_{j, t}^{(i)} 3^{n_{i}-t}
$$

The color assigned to vertex $x_{t}^{(i)}$ by the coloring $C_{j}^{(i)}$ is given by the coordinate $c_{j, t}^{(i)}$. A coloring $C_{j}^{(i)}$ is called non-valid if, for some $s, t \in\left\{1, \ldots, n_{i}\right\}$, we have $c_{j, s}^{(i)}=0$, $c_{j, t}^{(i)}=2$ and $\left(x_{s}^{(i)}, x_{t}^{(i)}\right) \in E$.

Table $A_{i}$, then, is of the form

$$
A_{i}=\left[\begin{array}{cc}
C_{1}^{(i)} & \min _{1}^{(i)} \\
\vdots & \vdots \\
C_{3^{n_{i}}}^{(i)} & \min _{3^{n_{i}}}^{(i)}
\end{array}\right]
$$

where $\min _{j}^{(i)} \in \mathbb{N} \cup\{+\infty\}$ is the variable which stores, at each stage of the dynamic programming step, the minimum number of vertices needed so far in a dominating set that uses the particular coloring $C_{j}^{(i)}$ for the vertices in $X_{i}$.

Now we are ready to describe the three steps in which the algorithm proceeds.

Step 1: In the first step of the algorithm, we initialize all tables $A_{i}$ as follows:

$$
\min _{j}^{(i)} \leftarrow\left\{\begin{array}{l}
+\infty, \quad \text { if the coloring represented by row } j \text { is non-valid } \\
\left|\left\{t \in\left\{1, \ldots, n_{i}\right\} \mid c_{j, t}^{(i)}=0\right\}\right|, \quad \text { otherwise }
\end{array}\right.
$$


Step 2: After this initialization, we visit the tables of our tree decomposition from the leaves to the root, updating the tables in each step as follows. Suppose node $i$ has a child $k$ in the tree $T$. We show how table $A_{i}$ is updated using table $A_{k}$. In the case that $i$ has several children $k_{1}, \ldots, k_{s}$ in the tree $T$, this step has to be repeated for each child.

1. Determine $X_{i} \cap X_{k}$, say $X_{i} \cap X_{k}=\left\{y_{1}, \ldots, y_{s}\right\}$.

2. W.l.o.g., we assume that $y_{1}=x_{1}^{(i)}=x_{1}^{(k)}, \ldots, y_{s}=x_{s}^{(i)}=x_{s}^{(k)}$. Otherwise we have to reorder the sets $X_{i}, X_{k}$ and the last column of the tables $A_{i}$ and $A_{k}$ accordingly. This can be done in $O\left(3^{\ell}\right)$ time as follows.

Suppose we have $y_{1}=x_{i_{1}}^{(i)}, \ldots, y_{s}=x_{i_{s}}^{(i)}$ and $\left\{1, \ldots, n_{i}\right\}=\left\{i_{1}, \ldots, i_{s}\right\} \cup$ $\left\{i_{s+1}, \ldots, i_{n_{i}}\right\}$. Then, we let

$$
X_{i} \leftarrow\left(x_{i_{1}}^{(i)}, \ldots, x_{i_{s}}^{(i)}, x_{i_{s+1}}^{(i)}, \ldots, x_{i_{n_{i}}}^{(i)}\right)
$$

and the corresponding table becomes

$$
A_{i} \leftarrow\left[\begin{array}{cc}
C_{1}^{(i)} & \min _{\phi(1)}^{(i)} \\
\vdots & \vdots \\
C_{3^{n_{i}}}^{(i)} & \min _{\phi\left(3^{n_{i}}\right)}^{(i)}
\end{array}\right], \quad \text { where } \phi(j)-1=\sum_{t=1}^{n_{i}} c_{j, i_{t}}^{(i)} 3^{n_{i}-t}
$$

The rearrangement for $X_{k}$ and the last column of $A_{k}$ has to be carried out similarly.

3. For each of the $3^{s}$ colorings $C=\left(c_{1}, \ldots, c_{s}\right) \in\{0,1,2\}^{s}$ of the vertices $y_{1}, \ldots, y_{s}$, we do the following.

(a) Determine

$$
\min (C)=\min \left\{\min _{j}^{(l)} \mid C_{j}^{(k)} \in C \times\{0,1\}^{n_{k}-s}\right\} .
$$

Note that, from property (3) of a tree decomposition (Definition 8), we know that none of the vertices $x_{s+1}^{(k)}, \ldots, x_{n_{k}}^{(k)}$ will appear in a bag that has not been visited up to this point. Otherwise, such a vertex would also appear in $X_{i}$. That is why, by evaluating $\min (C)$, we do not take into account the colorings that leave these vertices unresolvedly "white."

It takes $O\left(3^{\ell}\right)$ time to determine all these minima.

(b) For each of the row in $A_{i}$ that corresponds to a coloring $C_{j}^{(i)}$ of the form $C \times\{0,1\}^{n_{i}-s}$, we let

$$
\min _{j}^{(i)} \leftarrow \min _{j}^{(i)}+\min (C)-\left|\left\{t \in\{1, \ldots, s\} \mid c_{t}=0\right\}\right| .
$$


Again, all these assignments can be done in time $O\left(3^{\ell}\right)$.

Step 2 of the algorithm has running time $O\left(3^{\ell} N\right)$, where $N$ is the number of bags in the tree decomposition.

Step 3: Let $r$ denote the root of $T$. For the domination number $\gamma(G)$, we finally get

$$
\gamma(G)=\min \left\{\min _{j}^{(r)} \mid C_{j}^{(r)} \in\{0,1\}^{n_{r}}\right\}
$$

The total running time of the algorithm is $O\left(3^{\ell} N\right)$. For the correctness of the algorithm, we observe the following. Firstly, property (1) of a tree decomposition (see Definition 8) guarantees that each vertex is assigned a color. Secondly, in our initialization Step 1, we made sure that only valid colorings are taken into consideration. Since, by property (2) of a tree decomposition (see Definition 8), any pair of neighbors appears in at least one bag, the validity of the colorings was checked for each such pair of neighbors. And, thirdly, property (3) of a tree decomposition (see Definition 8), together with the comments after Step 2.2 of the algorithm, implies that the updating of each table is done consistently with all tables that have been visited earlier in the algorithm.

Also, note that, when bookkeeping how the minima in Step 2 were obtained, this algorithm constructs a dominating set $D$ corresponding to $\gamma(G)$.

We remark that Aspvall et al. [3] addressed the memory requirement problem arising in the type of algorithms described above. They suggested a method to minimize the sum of the sizes of the tables that need to be stored simultaneously.

Using Corollary 10 and Theorem 11, a straightforward solution to the PLANAR $k$-DOMINATING SET problem using tree decompositions leads to an algorithm which runs in time $O\left(3^{9 k} n\right)$. Downey and Fellows [21, 22] suggested an idea that leads to a faster search tree algorithm. They state an algorithm with running time $O\left(11^{k} n\right)$ (without using tree decompositions).

In section 3 , we show that a graph with a $k$-dominating set has treewidth $O(\sqrt{k})$. Moreover, we prove that a corresponding tree decomposition can be found in time $O(\sqrt{k} n)$. Combining this with Theorem 11 gives a significant asymptotic improvement of the result of Downey and Fellows.

\subsection{Treewidth and $r$-outerplanarity}

In this section, we give a constructive proof of the result stated in Theorem 9 . The proof is based upon the proof in [10, Theorem 83]. To be more precise, we prove:

Theorem 12 Let an r-outerplanar graph $G=(V, E)$ be given together with an $r$-outerplanar embedding. Then a tree decomposition $\left\langle\left\{X_{i} \mid i \in I\right\}, T\right\rangle$, with width at most $3 r-1$ and with $|I|=O(|V|)$ of $G$, can be found in $O(r|V|)$ time. 

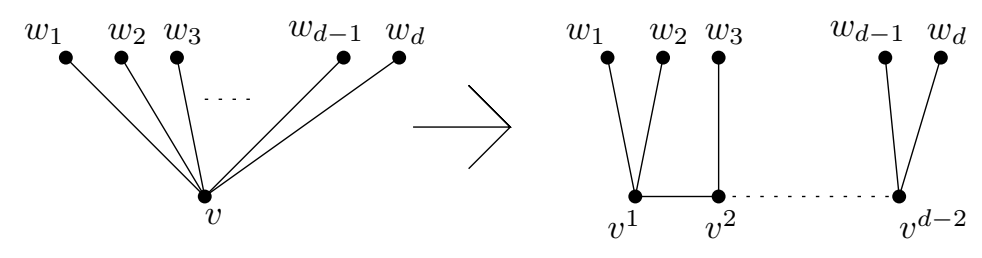

Figure 1: Replacing a vertex by a path with vertices of degree 3.

We make the assumption that with the embedding, for each vertex $v$, the incident edges of $v$ are given in clockwise order as they appear in the embedding. Most (linear time) graph planarity testing and embedding algorithms yield such orderings of the edge lists (see [18]).

Now, we discuss the proof of Theorem 12. For the construction of the desired tree decomposition we proceed in several steps. Firstly, we determine the layers of the given graph $G$. Secondly, $G$ is embedded in a graph $H$, whose degree is bounded by 3 and whose outerplanarity number doesn't exceed the one of $G$. Then, a suitable maximal spanning forest is constructed for $H$. This step is done inductively proceeding along the different layers of $H$ in a way that the so-called "edge and vertex remember numbers" are kept small. Using this spanning tree, in a further step, we determine a tree decomposition of $H$. Finally, this tree decomposition can be turned into a tree decomposition of $G$.

Determining the layers of the embedded graph. Without loss of generality, we assume $G$ is connected. Suppose $G$ is given with an $r$-outerplanar embedding with, for every vertex, a clockwise ordering of its adjacent edges. With the help of these orderings, one can build, in $O(|V|)$ time, the dual graph $G^{*}$, with pointers from edges of $G$ to the two adjacent faces in the dual. We can first partition the set of faces into 'layers': put a face $f$ in layer $L_{i+1}^{*}$ if the distance of this face in the dual graph to the exterior face is $i$. This distance can be determined in linear time using breadth-first search on the dual graph.

Now, a vertex $v$ of $G$ belongs to layer $L_{i}$ for the smallest $i$ such that $v$ is adjacent to a face $f_{v}$ in $L_{i}^{*}$. Note that faces can belong to layer $L_{r+1}^{*}$, but not to layers $L_{s}^{*}$ with $s>r+1$.

Embedding $G$ in a graph $H$ of degree three. The next step is to construct an $r^{\prime}$-outerplanar (where $\left.r^{\prime} \leq r\right)$ graph $H=\left(V_{H}, E_{H}\right)$ of degree at most three that contains $G$ as a minor, i.e., $G$ can be obtained from $H$ by a series of vertex deletions, edge deletions, and edge contractions.

In order to do this, every vertex with degree $d \geq 4$ is replaced by a path of $d-2$ vertices of degree 3, as shown in Fig. 1 . This is done in such a way that the graph stays $r^{\prime}$-outerplanar. More precisely, for each vertex $v$ of layer $L_{i}$ we determine the face $f_{v}$ in $L_{i}^{*}$ as described above. We then find two successive edges $\{x, v\},\{v, y\}$ that are border to $f_{v}$. Now, let $x$ take the role of $w_{1}$ and $y$ take the 
role of $w_{d}$ in Fig. 1. Observe that, in this manner, all vertices $v^{i}$ on the newly formed path are adjacent to face $f_{v} \in L_{i}^{*}$. Let $H$ be the graph obtained after replacing all vertices of degree at least four in this manner.

Note that $H$ has the same set of faces as $G$ (i.e., $H^{*}$ and $G^{*}$ share the same set of vertices) and that faces adjacent in $G$ are still adjacent in $H$ (i.e., $G^{*}$ is a subgraph of $H^{*}$ ). Hence, $H^{*}$ can be obtained from $G^{*}$ by possibly adding some further edges. Clearly, the minimum distance of a vertex in $H^{*}$ to the exterior face vertex may only decrease (not increase) compared to the corresponding distance in $G^{*}$. Since the layer to which a vertex belongs is exactly one more than the minimum distance of the adjacent faces to the exterior face, the outerplanarity number $r^{\prime}$ of $H$ is bounded by $r$.

Constructing a suitable maximal spanning forest for $H$. At this point, we have an $r^{\prime}$-outerplanar graph $H=\left(V_{H}, E_{H}\right)$ of maximum degree 3 . We now construct a maximal spanning forest $T$ for $H$ that yields small so-called "edge and vertex remember numbers." This step is done inductively along the different layers proceeding from inside towards the exterior.

Observe that when removing all edges on the exterior face of an s-outerplanar graph of maximum degree three, we obtain an $(s-1)$-outerplanar graph, when $s>1$. When we remove all edges on the exterior face of an outerplanar graph, we obtain a forest.

Thus, we can partition the edges into $r^{\prime}+1$ sets $E_{1}, \ldots, E_{r^{\prime}+1}$, with $E_{1}$ the edges on the exterior face, and $E_{i}$ the edges on the exterior face when all edges in $E_{1} \cup \ldots \cup E_{i-1}$ are removed. Again, using the dual, this partition can be computed in $O(|V|)$ time.

Now, we form a sequence of forests. We start with forest $T_{r^{\prime}+1}$, which consists of all edges in $E_{r^{\prime}+1}$. (Note that these are the interior edges of an outerplanar graph of maximum degree 3 , so $T_{r^{\prime}+1}$ is acyclic.)

When we have $T_{i}, 1<i \leq r^{\prime}+1$, we form $T_{i-1}$ in the following way: add a maximal set of edges from $E_{i-1}$ to $T_{i}$ such that no cycles are formed. Note that in this way, each $T_{i}$ is a maximal spanning forest of the subgraph formed by the edges in $E_{i} \cup \ldots \cup E_{r^{\prime}+1}$; we call this subgraph $H_{i}$. (Maximality is meant here with respect to set inclusion; a maximal spanning forest of an arbitrary graph hence can be found in $O(|V|)$ time using a standard depth first search approach, but here we need such a forest made in a specific way.)

It is not hard to see that one such step can be done in $O(|V|)$ time; as we do at most $r$ such steps, the time to build $T_{1}$ becomes $O(r|V|)$.

Definition 13 For a graph $G=(V, E)$, and a forest $T=(V, F)$ that is a subgraph of $G$, define the edge remember number $\operatorname{er}(G, T, e)$ of an edge $e \in F$ (with respect to $G$ and $T$ ) as the number of edges $\{v, w\} \in E-F$ such that there is a simple path in $T$ from $v$ to $w$ that uses $e$. The edge remember number of $T$ (with respect to $G$ ) is $\operatorname{er}(G, T)=\max _{e \in F} \operatorname{er}(G, T, e)$. The vertex remem- 
ber number $\operatorname{vr}(G, T, v)$ of a vertex $v \in V$ (with respect to $G$ and $T$ ) is the number of edges $\{v, w\} \in E-F$ such that there is a simple path in $T$ from $v$ to $w$ that uses $v$. The vertex remember number of $T$ (with respect to $G$ ) is $\operatorname{vr}(G, T)=\max _{v \in V} \operatorname{vr}(G, T, v)$.

One may observe that the construction of the trees $T_{i}$ is the one used in the proofs given in [10, Section 13]. The following result is proved in [10, Lemma 80]. Note that in order to obtain this result it is essential that the degree of $H$ is bounded by 3 :

Lemma 14 (i) For every $i, 1 \leq i \leq r^{\prime}+1$, er $\left(H_{i}, T_{i}\right) \leq 2\left(r^{\prime}+1-i\right)$.

(ii) For every $i, 1 \leq i \leq r^{\prime}, \operatorname{vr}\left(H_{i}, T_{i}\right) \leq 3\left(r^{\prime}+1-i\right)-1$.

Without loss of generality, we can suppose that $G$ and, therefore, $H$ is connected and, hence, we have a spanning tree $T_{1}$ of $H$ with $\operatorname{er}\left(H, T_{1}\right) \leq 2 r^{\prime}$ and $\operatorname{vr}\left(H, T_{1}\right) \leq 3 r^{\prime}-1$.

Deriving a tree decomposition from the spanning forest. We now apply the following result of [10, Theorem 71] to the graph $H$ and the spanning forest $T_{1}$ in order to obtain a tree decomposition in time $O(r|V|)$ of width bounded by

$$
\max \left(\operatorname{vr}\left(H, T_{1}\right), \operatorname{er}\left(H, T_{1}\right)+1\right) \leq 3 r^{\prime}-1 \leq 3 r-1 .
$$

For the sake of completeness of the whole construction, we outline the easy proof.

Theorem 15 Let $T=(V, F)$ be a maximal spanning forest for the graph $G=$ $(V, E)$. Then a tree decomposition with width at most $\max (\operatorname{vr}(G, T), \operatorname{er}(G, T)+1)$ and $O(|V|)$ nodes can be determined in $O(\operatorname{vr}(G, T) \cdot|V|)$ time.

Proof. Our aim is to construct a tree decomposition $\left\langle\left\{X_{i} \mid i \in I\right\}, T^{\prime}\right\rangle$ of $G$. Let $T^{\prime}=\left(V \cup F, F^{\prime}\right)$ with $F^{\prime}=\{\{v, e\} \mid v \in V, e \in F, \exists w \in V: e=\{v, w\}\}$ be the tree obtained by subdividing every edge of $T$. The bags $X_{i}$ for $i \in I:=V \cup F$ are obtained as follows. For every $v \in V$, add $v$ to $X_{v}$. For every $e=\{v, w\} \in F$, add $v$ and $w$ to $X_{e}$. Now, for every edge $e=\{v, w\}$ in $E$ but not in $F$, add $v$ to all sets $X_{u}$ and $X_{e}$, with $u \in V$ or $e \in F$ on the path from $v$ to $w$ in $T$.

Using standard graph algorithmic techniques, the shortest path between two vertices in a tree can be found in time proportional to the length of that path; since each vertex in $T$ can contribute to at most $\operatorname{vr}(G, T)$ such paths, the running time is bounded by $O(\operatorname{vr}(G, T) \cdot|V|)$.

It is easy to check that this indeed yields a tree decomposition. Its bags have size $\left|X_{v}\right| \leq 1+\operatorname{vr}(G, T)$ (for all $\left.v \in V\right)$ and $\left|X_{e}\right| \leq 2+\operatorname{er}(G, T)$ (for all $e \in E$ ). Hence, the resulting treewidth is at $\operatorname{most} \max (\operatorname{vr}(G, T), \operatorname{er}(G, T)+1)$.

Undoing the minor operations. Finally, the tree decomposition of $H$ can be turned into a tree decomposition of $G$ of equal or smaller width by replacing 
every occurrence of a vertex $v^{i}$ in a set $X_{i}$ by an occurrence of the corresponding vertex $v$ (see e.g., [10, Lemma 16].) This again costs time, linear in the total size of all sets $X_{i}$ in the tree decomposition, i.e., $O(r|V|)$ time.

Altogether this establishes the correctness of Theorem 12.

\section{The main result}

In this section, we show that a planar graph with domination number $k$ has treewidth of at most $O(\sqrt{k})$. This improves Corollary 10 considerably. In the next section, we will show how this new result can be turned into a constructive algorithm. Combining the results of this section with Theorem 11, we will present an algorithm having time complexity $3^{O(\sqrt{k})} n$. This obviously gives an asymptotic improvement of the $O\left(11^{k} n\right)$ algorithm presented by Downey and Fellows [21, 22].

This section is organized as follows. In a first subsection, we make some general observations on how to construct tree decompositions using separators. The following two subsections show that, in a planar graph which admits a $k$ dominating set, we can find small separators layerwise. Finally, the results are pieced together to prove our main result in this section.

\subsection{Separators and treewidth}

Here, the main idea is to find small separators of the graph and to merge the tree decompositions of the resulting subgraphs.

Definition 16 Let $G=(V, E)$ be a graph. A subset $S$ of the vertex set $V$ is called a separator of $G$, if the subgraph $G-S$ is disconnected.

For any given separator splitting a graph into different components, we obtain a simple upper bound for the treewidth of this graph which depends on the size of the separator and the treewidth of the resulting components.

Proposition 17 If a connected graph can be decomposed into components of treewidth of at most $t$ by means of a separator of size $s$, then the whole graph has treewidth of at most $t+s$.

Proof. The separator splits the graph into different components. Suppose we are given the tree decompositions of these components of width at most $t$. The goal is to construct a tree decomposition for the original graph. This can be achieved by firstly merging the separator to every bag in each of these given tree decompositions. In a second step, add some arbitrary connections between the trees corresponding to the components. It is straightforward to check that this forms a tree decomposition of the whole graph of width at most $t+s$.

For plane graphs, there is an iterated version of this observation. 
Proposition 18 Let $G$ be a plane graph with layers $L_{i},(i=1, \ldots, r)$. For $i=1, \ldots, \ell$, let $\mathcal{L}_{i}$ be a set of consecutive layers, i.e., $\mathcal{L}_{i}=\left\{L_{j_{i}}, L_{j_{i}+1}, \ldots, L_{j_{i}+n_{i}}\right\}$, such that $\mathcal{L}_{i} \cap \mathcal{L}_{i^{\prime}}=\emptyset$ for all $i \neq i^{\prime}$. Moreover, suppose $G$ can be decomposed into components, each of treewidth of at most $t$, by means of separators $S_{1}, \ldots, S_{\ell}$, where $S_{i} \subseteq \bigcup_{L \in \mathcal{L}_{i}} L$ for all $i=1, \ldots, \ell$. Then $G$ has treewidth of at most $t+2 s$, where $s=\max _{i=1, \ldots, \ell}\left|S_{i}\right|$.

Proof. The proof again uses the merging-techniques illustrated in the previous proposition: Suppose, w.l.o.g., the sets $\mathcal{L}_{i}$ appear in successive order, i.e., $j_{i}<$ $j_{i+1}$. For each $i=0, \ldots, \ell$, consider the component $G_{i}$ of treewidth at most $t$ which is cut out by the separators $S_{i}$ and $S_{i+1}$ (by default, we set $S_{0}=S_{\ell+1}=\emptyset$ ). We add $S_{i}$ and $S_{i+1}$ to every node in a given tree decomposition of $G_{i}$. In order to obtain a tree decomposition of $G$, we successively add an arbitrary connection between the trees $T_{i}$ and $T_{i+1}$ of the so-modified tree decompositions that correspond to the subgraphs $G_{i}$ and $G_{i+1}$.

\subsection{Finding separators layerwise}

In the following, we assume that our graph $G$ has a fixed plane embedding with $r$ layers. We show that the treewidth cannot exceed $O(\sqrt{k})$ if a dominating set of size $k$ exists.

We assume that we have a dominating set $D$ of size at most $k$. Let $k_{i}$ be the number of vertices of $D_{i}=D \cap L_{i}$. Hence, $\sum_{i=1}^{r} k_{i}=k$. In order to avoid case distinctions, we set $k_{0}=k_{r+1}=k_{r+2}=0$. Moreover, let $c_{i}$ denote the number of non-vacuous layer-components of layer $L_{i}$.

Our approach is based on finding small separators in $G$ layerwise. More precisely, for each $i=1, \ldots, r-2$, we want to construct a set $S_{i} \subseteq L_{i-1} \cup L_{i} \cup L_{i+1}$ separating layer $L_{i-1}$ from layer $L_{i+2}$ in such a way ${ }^{1}$ that the total size of these sets can be bounded by some linear term in $k$. The key idea for proving that $S_{i}$ separates layers $L_{i-1}$ from $L_{i+2}$ relies on a close investigation of the paths leaving layer $L_{i-1}$ to the interior of the graph. Each such path passes a "first" vertex in layer $L_{i}$. This particular vertex can be dominated by vertices from $D_{i-1}, D_{i}$, or $D_{i+1}$. It turns out that, in order to cut this particular path, the set $S_{i}$ has to contain the vertices of the sets $D_{i-1}, D_{i}$, and $D_{i+1}$ plus some suitably chosen pairs of neighbors of any of these vertices. This results in letting $S_{i}$ be the union of so-called "upper," "lower," and "middle" triples. We will carry out the to some extent technically complicated step in what follows.

For this purpose, in the following, we write $N(x)$ to describe the set of neighbors of a vertex $x$ and use the notion $B(\cdot)$ for boundary cycles as introduced in Definition 7 .

\footnotetext{
${ }^{1}$ Intuitively, one might be tempted to construct a set of vertices separating layer $L_{i-1}$ from layer $L_{i+1}$. However, for technical reasons that become clear in the proof of the forthcoming Theorem 23, it is more convenient to separate layers $L_{i-1}$ and $L_{i+2}$.
} 


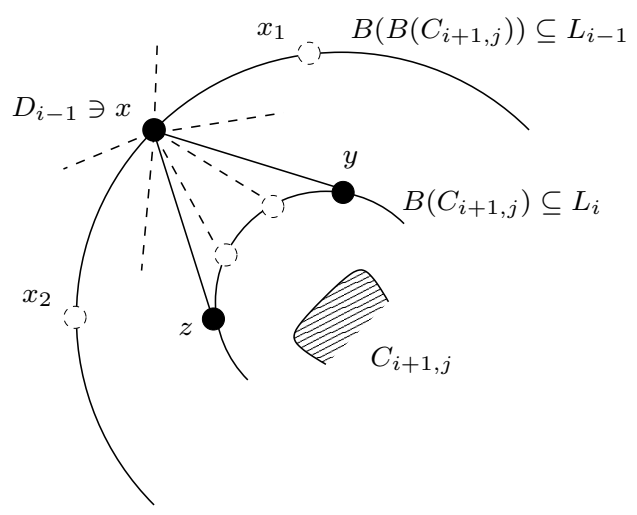

Figure 2: Upper triples.

Upper triples. An upper triple for layer $L_{i}$ is associated to a non-vacuous ${ }^{2}$ layer-component $C_{i+1, j}$ of layer $L_{i+1}$ and a vertex $x \in D_{i-1}$ that has a neighbor on the boundary cycle $B\left(C_{i+1, j}\right)$ (see Fig. 2). Then, clearly, $x \in B\left(B\left(C_{i+1, j}\right)\right)$, by definition of a boundary cycle. Let $x_{1}$ and $x_{2}$ be the neighbors of $x$ on the boundary cycle $B\left(B\left(C_{i+1, j}\right)\right)$. Starting from $x_{1}$, we go around $x$ up to $x_{2}$ so that we visit all neighbors of $x$ in layer $L_{i}$. We note the neighbors of $x$ on the boundary cycle $B\left(C_{i+1, j}\right)$. Going around gives two outermost neighbors $y$ and $z$ on this boundary cycle. The triple, then, is the three-element set $\{x, y, z\}$. In case $x$ has only a single neighbor $y$ in $B\left(C_{i+1, j}\right)$, the "triple" consists of only $\{x, y\}$ (let, by default, $z=y$ ).

Definition 19 For each non-vacuous layer-component $C_{i+1, j}$ of $L_{i+1}$ and each vertex $x \in D_{i-1}$ with neighbors in $B\left(C_{i+1, j}\right)$, the set $\{x, y, z\}$ as described above is called an upper triple of layer $L_{i}$.

Lower triples. A lower triple for layer $L_{i}$ is associated to a vertex $x \in D_{i+1}$ and a non-vacuous layer-component $C_{i+1, j}$ of layer $L_{i+1}$ (see Fig. 3). We only consider layer-components $C_{i+1, j}$ of layer $L_{i+1}$ that are enclosed by the boundary cycle $B(\{x\}$ ). For each pair $\tilde{y}, \tilde{z} \in B(\{x\}) \cap N(x)$ (where $\tilde{y} \neq \tilde{z}$ ), we consider the path $P_{\tilde{y}, \tilde{z}}$ from $\tilde{y}$ to $\tilde{z}$ along the cycle $B(\{x\})$, taking the direction such that the

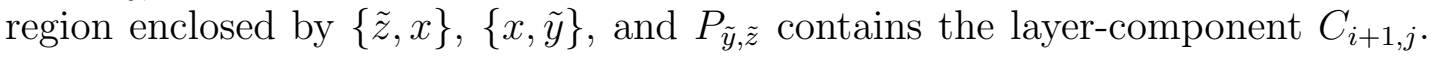
Let $\{y, z\} \subseteq B(\{x\}) \cap N(x)$ be the pair such that the corresponding path $P_{y, z}$ is shortest. The triple, then, is the three-element set $\{x, y, z\}$. If $x$ has no or only a single neighbor $y$ in $B(\{x\})$, then the "triple" consists only of $\{x\}$, or $\{x, y\}$ (by default, in these cases, we let $x=y=z$, or $y=z$, respectively).

\footnotetext{
${ }^{2}$ Note that here, as well as in the definitions of middle and upper triples, all vacuous components of layer $L_{i+1}$ are of no interest to us, since we want to find a set of vertices separating levels $L_{i-1}$ from $L_{i+2}$.
} 


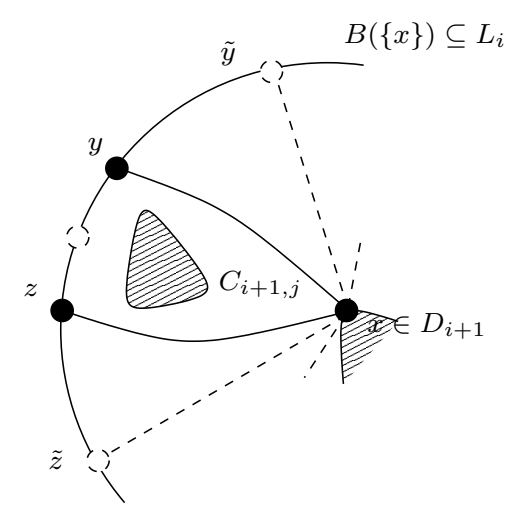

Figure 3: Lower triples.

Definition 20 For each vertex $x \in C_{i+1, j}$ of $D_{i+1}$ and each non-vacuous layercomponent $C_{i+1, j}$ that is enclosed by $B(\{x\})$, the set $\{x, y, z\}$ as described above is called a lower triple of layer $L_{i}$.

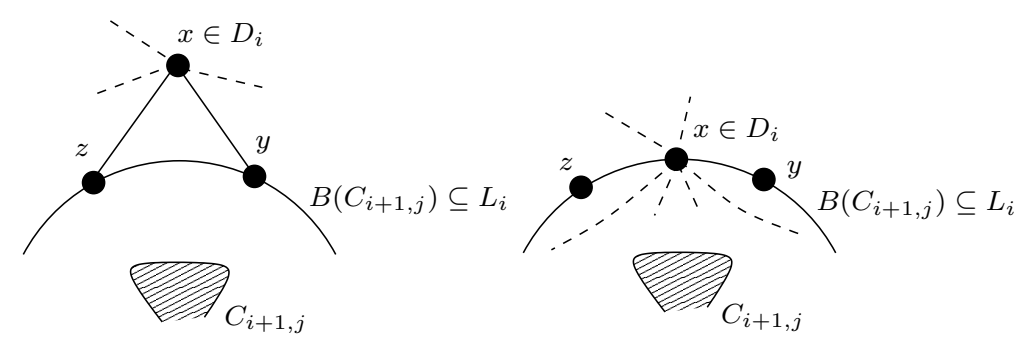

Case 1:

Case 2:

Figure 4: Middle triples.

Middle triples. A middle triple for layer $L_{i}$ is associated to a non-vacuous layer-component $C_{i+1, j}$ and a vertex $x \in D_{i}$ that has a neighbor in $B\left(C_{i+1, j}\right)$ (see Fig. 4). Note that, due to the layer model, it is easy to see that a vertex $x \in D_{i}$ can have at most two neighbors $y, z$ in $B\left(C_{i+1, j}\right)$. Depending on whether $x$ itself lies on the cycle $B\left(C_{i+1, j}\right)$ or not, we obtain two different cases which are both illustrated in Fig. 4. In either of these cases, the middle triple is defined as the set $\{x, y, z\}$. Again, if $x$ has none or only a single neighbor $y$ in $B\left(C_{i+1, j}\right)$, then the "triple" consists only of $\{x\}$, or $\{x, y\}$, respectively (by default, in these cases, we let $x=y=z$, or $y=z$, respectively).

Definition 21 For each non-vacuous layer-component $C_{i+1, j}$ and each vertex $x \in D_{i}$, the set $\{x, y, z\}$ as described above is called a middle triple for layer $L_{i}$. 


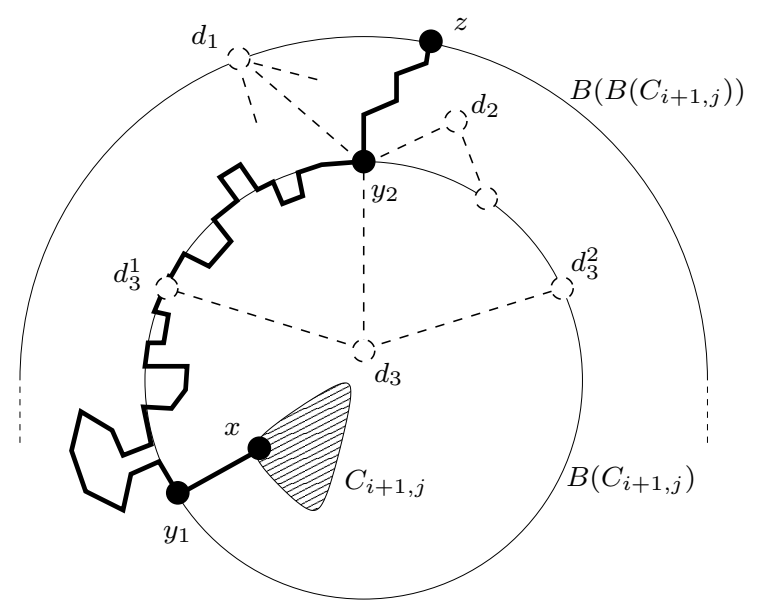

Figure 5: $S_{i}$ separates $L_{i-1}$ and $L_{i+2}$.

Definition 22 We define the set $S_{i}$ as the union of all upper triples, lower triples and middle triples of layer $L_{i}$.

In the following, we will show that $S_{i}$ is a separator of the graph. Note that the upper bounds on the size of $S_{i}$, which are derived afterwards, are crucial for the upper bound on the treewidth derived later on.

Theorem 23 The set $S_{i}$ separates vertices of layers $L_{i-1}$ and $L_{i+2}$.

Proof. Suppose there is a path $P$ (with no repeated vertices) from layer $L_{i+2}$ to layer $L_{i-1}$ that avoids $S_{i}$. This clearly implies that there exists a path $P^{\prime}$ from a vertex $x$ in a non-vacuous layer-component $C_{i+1, j}$ of layer $L_{i+1}$ to a vertex $z \in B\left(B\left(C_{i+1, j}\right)\right)$ in layer $L_{i-1}$ which has the following two properties:

- $P^{\prime} \cap S_{i}=\emptyset$.

- All vertices inbetween $x$ and $z$ belong to layer $L_{i}$ or to vacuous layercomponents of layer $L_{i+1}$.

This can be achieved by simply taking a suitable subpath $P^{\prime}$ of $P$. Let $y_{1}$ (and $y_{2}$, respectively) be the first (last) vertex along the path $P^{\prime}$ from $x$ to $z$ that lies on the boundary cycle $B\left(C_{i+1, j}\right) \subseteq L_{i}$ (see Fig. 5).

Obviously, $y_{2}$ cannot be an element of $D$ since, then, it would appear in a middle triple of layer $L_{i}$ and, hence, in $S_{i}$. We now consider the vertex that dominates $y_{2}$. This vertex can lie in layer $L_{i-1}, L_{i}$, or $L_{i+1}$.

Suppose first that $y_{2}$ is dominated by a vertex $d_{1} \in L_{i-1}$. Then $d_{1}$ is in $B\left(B\left(C_{i+1, j}\right)\right.$ ), simply by definition of the boundary cycle (see Fig. 5). Since $G$ is planar, this implies that $y_{2}$ must be an "outermost" neighbor of $d_{1}$ among all elements in $N\left(d_{1}\right) \cap B\left(C_{i+1, j}\right)$. If this were not the case, then there would be 
an edge from $d_{1}$ to a vertex on $B\left(C_{i+1, j}\right)$ that leaves the closed region bounded by $\left\{d_{1}, y_{2}\right\}$, the path from $y_{2}$ to $z$, and the corresponding path from $z$ to $d_{1}$ along $B\left(B\left(C_{i+1, j}\right)\right)$. Hence, $y_{2}$ would be in the upper triple of layer $L_{i}$ which is associated to the layer-component $C_{i+1, j}$ and $d_{1}$. This contradicts the fact that $P^{\prime}$ avoids $S_{i}$.

Now, suppose that $y_{2}$ is dominated by a vertex $d_{2} \in D_{i}$ (see Fig. 5). By definition of the middle triples, this clearly implies that $y_{2}$ is in the middle triple associated to $C_{i+1, j}$ and $d_{2}$. Again, this contradicts the assumption that $P^{\prime} \cap S_{i}=$ $\emptyset$.

Consequently, the dominating vertex $d_{3}$ of $y_{2}$ has to lie in layer $L_{i+1}$. Let $\left\{d_{3}, d_{3}^{1}, d_{3}^{2}\right\}$, where $d_{3}^{1}, d_{3}^{2} \in N\left(d_{3}\right) \cap B\left(C_{i+1, j}\right)$, be the lower triple associated to layer-component $C_{i+1, j}$ and $d_{3}$ (see Fig. 5). By definition, $C_{i+1, j}$ is contained in the region enclosed by $\left\{d_{3}^{1}, d_{3}\right\},\left\{d_{3}, d_{3}^{2}\right\}$, and the path from $d_{3}^{2}$ to $d_{3}^{1}$ along $B\left(C_{i+1, j}\right)$, which - assuming that $y_{2} \notin\left\{d_{3}, d_{3}^{1}, d_{3}^{2}\right\}$ - does not hit $y_{2}$ (see Fig. 5). We now observe that, whenever the path from $y_{1}$ to $y_{2}$ leaves the cycle $B\left(C_{i+1, j}\right)$ to its exterior, say at a vertex $q$, then it has to return to $B\left(C_{i+1, j}\right)$ at a vertex $q^{\prime} \in N(q) \cap B\left(C_{i+1, j}\right)$. Otherwise, we would violate the fact that, by definition, $B\left(C_{i+1, j}\right) \subseteq L_{i}$. This, however, shows that the path $P^{\prime}$ has to hit either $d_{3}^{1}$ or $d_{3}^{2}$ on its way from $y_{1}$ to $y_{2}$. Since $d_{3}^{1}, d_{3}^{2} \in S_{i}$, this case also contradicts the fact that $P^{\prime} \cap S_{i}=\emptyset$.

\subsection{An upper bound for the size of the separators}

In this subsection, we want to show that the sum of the cardinalities of all separators can be bounded by some linear term in $k$.

Lemma $24\left|S_{i}\right| \leq 5\left(k_{i-1}+k_{i}+k_{i+1}\right)+12 c_{i+1}$.

Proof. We give bounds for the number of vertices in upper, middle, and lower triples of layer $i$, separately.

Firstly, we discuss the upper triples of layer $i$, which were associated to a non-vacuous layer-component $C_{i+1, j}$ of layer $L_{i+1}$ and a vertex $x \in D_{i-1}$ with neighbors in $B\left(C_{i+1, j}\right)$. Consider the bipartite graph $G^{\prime}$ which has vertices for each non-vacuous layer-component $C_{i+1, j}$ and for each vertex in $D_{i-1}$. Whenever a vertex in $D_{i-1}$ has a neighbor in $B\left(C_{i+1, j}\right)$, an edge is drawn between the corresponding vertices in $G^{\prime}$. Each edge in $G^{\prime}$, by construction, may correspond to an upper triple of layer $L_{i}$. Note that $G^{\prime}$ is a planar bipartite graph whose bipartition subsets consist of $k_{i-1}$ and $c_{i+1}$ vertices, respectively. Thus, the number of edges of $G^{\prime}$ is linear in the number of vertices; more precisely, it is bounded by $2\left(k_{i-1}+c_{i+1}\right)$ (see [43, Corollary 1.2.]). From this, we obtain an upper bound for the number of vertices in upper triples of layer $L_{i}$ as follows: Potentially, each vertex of $D_{i-1}$ appears in an upper triple and, for each edge in $G^{\prime}$, we possibly 
obtain two further vertices in an upper triple. This shows that the total number of vertices in upper triples is bounded by $k_{i-1}+4\left(k_{i-1}+c_{i+1}\right)$.

A similar analysis can be used to show that the number of vertices in the lower triples is bounded by $k_{i+1}+4\left(k_{i+1}+c_{i+1}\right)$ and that the number of vertices in the middle triples can be bounded by $k_{i}+4\left(k_{i}+c_{i+1}\right)$.

By definition of $S_{i}$, this proves our claim.

Note that, by a more detailed investigation, the bound given in Lemma 24 probably can be improved. One observes, e.g., that the planar bipartite graph $G^{\prime}$, which was constructed in the proof, has the special property that it is a "hyperplane" bipartite graph, i.e., one of the bipartition subsets can be arranged on a line and all edges of the graph lie in one halfplane of this line. This property of $G^{\prime}$ is immediate from the fact that the upper triples associated to a non-vacuous layer-component $C_{i+1, j}$ lie within the boundary cycle $B\left(B\left(C_{i+1, j}\right)\right)$. For such graphs, our investigations indicate that one might obtain better estimates on the number of their edges than the ones used in the proof of Lemma 24.

A similar observation can be made for estimating the bounds for the lower triples.

For the number $c_{i}$ of non-vacuous components in layer $i$ we have the following estimate.

Lemma $25 c_{i} \leq k_{i}+k_{i+1}+k_{i+2}$.

Proof. By definition, $c_{i}$ refers to only non-vacuous layer-components in layer $L_{i}$, i.e., there is at least one vertex of layer $L_{i+1}$ contained within each such layercomponent. Such a vertex can only be dominated by a vertex from layer $L_{i}, L_{i+1}$, or $L_{i+2}$. In this way, we get the claimed upper bound.

Combining the two previous results yields the bound claimed at the beginning of this subsection.

Proposition $26 \sum_{i=1}^{r}\left|S_{i}\right| \leq 51 k$, where $r$ is the number of layers of the graph.

Proof. This follows directly when we combine the previous two lemmas, noting that $\sum_{i=1}^{r} k_{i}=k$.

\subsection{An improved relation of domination number and treewidth}

We are now ready to state our main result relating the domination number and the treewidth of a planar graph. Note that the following theorem gives a decisive asymptotic improvement of Corollary 10.

Theorem 27 A planar graph with domination number $k$ has treewidth of at most $6 \sqrt{34} \sqrt{k}+8$. 
Proof. Using the separators $S_{i}$ as found in the previous subsections, we consider the following three sets of vertices: $\mathbb{S}_{1}=S_{1} \cup S_{4} \cup S_{7} \cup \ldots, \mathbb{S}_{2}=S_{2} \cup S_{5} \cup S_{8} \cup \ldots$, and $\mathbb{S}_{3}=S_{3} \cup S_{6} \cup S_{9} \cup \ldots$. Since, by Proposition 26, $\left|\mathbb{S}_{1}\right|+\left|\mathbb{S}_{2}\right|+\left|\mathbb{S}_{3}\right| \leq 51 k$, one of these sets has size at most $\frac{51}{3} k$, say $\mathbb{S}_{\delta}$ (with $\delta \in\{1,2,3\}$ ).

Let $\delta$ and $\mathbb{S}_{\delta}$ be obtained as above. Let $d:=\frac{3}{2} \sqrt{34}$. We now go through the sequence $S_{1+\delta}, S_{4+\delta}, S_{7+\delta}, \ldots$ and look for separators of size at most $s(k):=d \sqrt{k}$. Due to the estimate on the size of $\mathbb{S}_{\delta}$, such separators of size at most $s(k)$ must appear within each $n(k):=\frac{51}{3} \cdot \frac{1}{d \sqrt{k}} \cdot k=\frac{1}{3} \sqrt{34} \sqrt{k}$ sets in the sequence. In this manner, we obtain a set of disjoint separators of size at most $s(k)$ each, such that any two consecutive separators from this set are at most $3 n(k)$ layers apart. Clearly, the separators chosen in this way fulfill the requirements in Proposition 18.

Observe that the components cut out in this way each have at most $3(n(k)+1)$ layers and, hence, their treewidth is bounded by $9(n(k)+1)-1$ due to Theorem 9 .

Using Proposition 18, we can compute an upper bound on the treewidth tw of the originally given graph with domination number $k$ :

$$
\begin{aligned}
\operatorname{tw}(k) & \leq 2 s(k)+9(n(k)+1)-1 \\
& =2\left(\frac{3}{2} \sqrt{34} \sqrt{k}\right)+9\left(\frac{1}{3} \sqrt{34} \sqrt{k}\right)+8 \\
& =6 \sqrt{34} \sqrt{k}+8 .
\end{aligned}
$$

This proves our claim.

How did we come to the constants? We simply computed the minimum of $2 s(k)+9(n(k)+1)-1$ (the upper bound on the treewidth) given the bound $s(k) n(k) \leq \frac{51}{3} k$. This suggests $s(k)=d \sqrt{k}$, and $d$ is optimal when $2 s(k)=$ $9 n(k)=9 \cdot \frac{51}{3} \cdot k \cdot s(k)^{-1}$, so, $2 d=\frac{153}{d}$, i.e., $d=\frac{3}{2} \sqrt{34}$.

Observe that the tree structure of the tree decomposition obtained in the preceding proof corresponds to the structure of the layer decomposition forest.

\section{The algorithm}

In this section, we outline our fixed parameter algorithm for solving PLANAR $k$ DOMINATING SET constructively. The input instance to the algorithm is a planar graph $G$ and a positive integer $k$. The algorithm determines whether $G$ admits a dominating set of size at most $k$, and, if so, constructs such a set. The running time for the algorithm will be $3^{O(\sqrt{k})} n$, where $n=|V|$.

Clearly, this generalizes to constructing a minimum size dominating set in a plane graph in time $3^{O(\sqrt{\gamma(G)})} n$, where $\gamma(G)$ is the domination number of the graph. If $\gamma(G)$ is not known in advance, one has to apply the fixed parameter 
algorithm and try different values of $k$ - this can be done at the cost of an extra multiplicative factor of $O(\log \gamma(G))$ by using binary search.

The key idea for our algorithm is to construct a tree decomposition of the stated width. However, Theorem 27 is a pure existence theorem which cannot be made constructive directly. There is one point that needs specific attention. As we do not start with the dominating set given, we cannot construct the upper, middle, and lower triples. Instead, we have to compute the minimum size separator $\hat{S}_{i}$ between $L_{i-1}$ and $L_{i+2}$ directly, and use that set instead of $S_{i}$ as defined in the proof of Section 3.2. Such a minimum size separator can be computed with well known techniques based on maximum flow (see, e.g., [33]).

Our algorithm proceeds in the following steps:

1. Embed the planar graph $G=(V, E)$ crossing-free into the plane. Determine the outerplanarity number $r$ of this embedding and all layers $L_{1}, \ldots, L_{r}$. By default, we set $L_{i}=\emptyset$ for all $i<0$ and $i>r$.

2. If $r>3 k$ then exit (there exists no $k$-dominating set). This step of the algorithm is justified by Proposition 4 .

3. For $\delta=1,2,3$ and $i=0, \ldots,\left\lfloor\frac{r}{3}\right\rfloor-1$, find the minimum separator $\hat{S}_{3 i+\delta}$, separating layers $L_{(3 i+\delta)-1}$ and $L_{(3 i+\delta)+2}$. Let $s_{3 i+\delta}=\left|\hat{S}_{3 i+\delta}\right|$.

4. Check whether there exists a $\delta \in\{1,2,3\}$ and an increasing sequence $\left(i_{j}\right)_{j=1, \ldots, t}$ of indices in $\left\{0, \ldots,\left\lfloor\frac{r}{3}\right\rfloor-1\right\}$, such that

$$
\begin{aligned}
s_{3 i_{j}+\delta} & \leq s(k):=\frac{3}{2} \sqrt{34} \sqrt{k} \text { for all } j=1, \ldots, t \text { and } \\
\left|i_{j+1}-i_{j}\right| & \leq n(k):=\frac{1}{3} \sqrt{34} \sqrt{k} \text { for all } j=1, \ldots, t-1 .
\end{aligned}
$$

If the answer is "no", then exit (there exists no $k$-dominating set). This step of the algorithm is justified by the considerations in the proof of Theorem 27.

5. Consider the separators $\mathcal{S}_{j}:=\hat{S}_{3 i_{j}+\delta}$ for $j=1, \ldots, t$ (by default, $\mathcal{S}_{j}=\emptyset$ for all other $j$ ) and, for each $j=0, \ldots, t$, let $G_{j}$ be the subgraph cut out by the separators $\mathcal{S}_{j}$ and $\mathcal{S}_{j+1}$ or, more precisely, let

$$
G_{j}:=G-\left(\bigcup_{\ell=\left(3 i_{j}+\delta\right)-1}^{\left(3 i_{(j+1)}+\delta\right)+1} L_{\ell} \backslash\left(\mathcal{S}_{j} \cup \mathcal{S}_{j+1}\right)\right) .
$$

Note that $G_{j}$ is at most $3(n(k)+1)$-outerplanar. 
6. Construct tree decompositions $\mathcal{X}_{j}$ for $G_{j}(j=0, \ldots, t)$ with $O(n)$ nodes each. For this step, we refer to Theorem 12.

7. Construct a tree decomposition $\mathcal{X}$ of $G$ with $O(n)$ nodes using the separators $\mathcal{S}_{1}, \ldots, \mathcal{S}_{t}$ and the tree decompositions $\mathcal{X}_{j}$ by the separator merging technique described in the proof of Proposition 18.

8. Solve the DOminating SET problem for $G$ with tree decomposition $\mathcal{X}$ as described in Theorem 11.

Step 1 can be implemented with methods described in Section 2.3.

As to Step 3 of the algorithm, we want to remark that such a minimum size separator can be computed with well known techniques based on maximum flow (see, e.g., [33]) as follows: For given values of $i$ and $\delta$, we first, build the graph $G^{\prime}$, induced by the vertices in $L_{3 i+\delta-1} \cup L_{3 i+\delta} \cup L_{3 i+\delta+1} \cup L_{3 i+\delta+2}$. Then, we contract all vertices in $L_{3 i+\delta-1}$ to one vertex $s$, and all vertices in $L_{3 i+\delta+2}$ to one vertex $t$. We look for a minimum $s$-t-separator in the resulting graph but, if this separator has size more than $s(k)$, then we just note that no separator of size at most $s(k)$ exists (compare with Step 4). Finding the separator or determining that no small enough separator exists can be done with the following standard method. Build a directed graph $G^{\prime \prime}$ in the following way. Every vertex $v$ in the graph is replaced by two vertices $v^{-}$and $v^{+}$, with an edge from $v^{-}$to $v^{+}$, and an edge $\{v, w\}$ is replaced by two edges $\left(v^{+}, w^{-}\right),\left(w^{+}, v^{-}\right)$. As explained in [38, Lemma 11, page 83], the minimum $s-t$ separator in $G^{\prime}$ can be found by applying a maximum flow algorithm in $G^{\prime \prime}$. To keep the running time in the stated bounds, we use the FordFulkerson maximum flow algorithm, but stop as soon as a flow value of more than $s(k)$ is used since, in such a case, we can conclude that no $s$-t-separator of size at most $s(k)$ exists. As each flow augmentation step costs time linear in the number of vertices and edges of $G^{\prime \prime}$ and increases the flow value by one, the time used by this procedure is $O\left(n^{\prime} \cdot s(k)\right)$, with $n^{\prime}=\left|L_{3 i+\delta-1} \cup L_{3 i+\delta} \cup L_{3 i+\delta+1} \cup L_{3 i+\delta+2}\right|$.

As for every vertex in $G$, there are at most four combinations of $\delta$ and $i$ in Step 3 such that it belongs to $L_{3 i+\delta-1} \cup L_{3 i+\delta} \cup L_{3 i+\delta+1} \cup L_{3 i+\delta+2}$ and the total time of step 3 is bounded by $O(|V| \cdot s(k))=O(n \sqrt{k})$.

The correctness of the algorithm above follows from our considerations in the previous sections.

Steps 1-7 allow us to construct a tree decomposition of width $6 \sqrt{34} \sqrt{k}+9$ of $G$ in $O(\sqrt{k} n)$ time. The running time for the last step in the algorithm is $O\left(3^{6 \sqrt{34} \sqrt{k}} n\right)$.

Summarizing these observations, we obtain the following theorem.

Theorem 28 The PLANAR $k$-DOMINATING SET problem can be solved in time $O\left(c^{\sqrt{k}} n\right)$, where $n$ is the size of the graph $G$, and $c=3^{6 \sqrt{34}}$. Moreover, if $\gamma(G) \leq$ $k$, a minimum size dominating set can be constructed within the same time. 
The constant $c$ above is $3^{6 \sqrt{34}}$, which is rather large. However, a more refined analysis should help to reduce this constant. Moreover, it is a worst case estimate, which might be far from what happens in practical applications.

We want to mention that an algorithm of similar running time could be obtained by combining our result (Theorem 27) with the $O\left(8^{r} n\right)$ time algorithm for solving DOMINATING SET on $r$-outerplanar graphs indicated by Baker [5]. To that end, one executes Steps 1-5 as presented above. Afterwards Steps 6-8 are replaced by a dynamic programming approach, the idea of which we briefly want to sketch. One keeps tables $A_{i}$ for each of the separators $\mathcal{S}_{i}$ similar to the algorithm presented in the proof of Theorem 11. Again, one uses three distinct colors for the vertices in the separator $\mathcal{S}_{i}$ :

- "black" (meaning, that the vertex belongs to the dominating set)

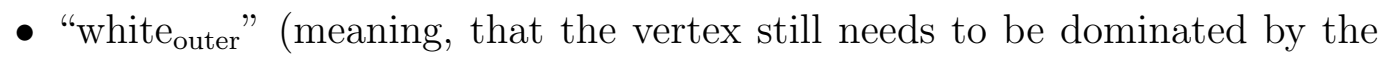
subgraph $G_{i-1}$ )

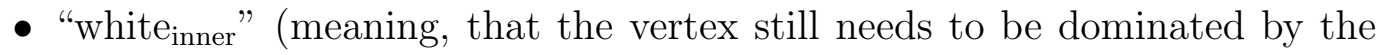
subgraph $G_{i}$ )

The tables are updated starting from $i=1$ up to $i=t$ as follows. The new entry for a specific coloring $C^{(i+1)}$ in table $A_{i+1}$ can be obtained from minimizing over all colorings $C^{(i)}$ in table $A_{i}$ and applying Baker's algorithm to the graph $G_{i}$ precolored according to $C^{(i)}$ and $C^{(i+1)}$. Working out the details of this approach

yields a running time of $O\left(c^{\sqrt{k}} n\right)$ for some slightly better constant $c$ than the one obtained in Theorem 28. However, we want to point out that the algorithm claimed by Baker is not worked out in detail for the DOMINATING SET problem, and, hence, it is hard to verify whether it can deal with precolored graphs, a basic assumption we need for this approach.

\section{Variations of PLANAR DOMINATING SET and the FACE COVER problem}

For several variations of the PLANAR DOMINATING SET problem, our technique can also help to obtain algorithms with a similar running time. In particular, we will consider the following problems: DOMINATING SET WITH PROPERTY $P$, PLANAR WEIGHTED DOMINATING SET, and FACE COVER.

\subsection{Planar dominating Set With Property $P$}

In the following, a property $P$ of a vertex set $V^{\prime} \subseteq V$ of an undirected graph $G=(V, E)$ will be a Boolean predicate which yields true or false values when 
given as input $V, E$, and $V^{\prime}$. Since $V$ and $E$ will always be clear from the context, we will simply write $P\left(V^{\prime}\right)$ instead of $P\left(V, E, V^{\prime}\right)$. Examples for such properties $P$ are:

- $V^{\prime}$ is an independent set, i.e., the graph induced by $V^{\prime}$ has no edges, or

- the graph induced by $V^{\prime}$ is connected.

A $k$-dominating set with property $P$ of an undirected graph $G$ is a $k$-dominating set $D$ of $G$ which has the additional property $P(D)$ in $G$. The DOMinating SET WITH PROPERTY $P$ problem is the task to find a minimum size dominating set with property $P$. The $k$-DOMINATING SET With PROPERTY $P$ problem is the task to decide, given a graph $G=(V, E)$, a property $P$, and a positive integer $k$, whether or not there exists a $k$-dominating set with property $P$.

Examples for such problems are:

- the $k$-indePendent Dominating SET problem, see [47, 48] or [22, p.464], where the property $P(D)$ of the $k$-dominating set $D$ is that $D$ is independent,

- the $k$-TOTAL DOMinating SET problem, see [47, 48], where the property $P(D)$ of the $k$-dominating set $D$ is that each vertex of $D$ has a neighbor in $D$,

- the $k$-PERfECT DOMINATING SET problem [47, 48], where the property $P(D)$ of the $k$-dominating set $D$ is that each vertex which is not in $D$ has exactly one neighbor in $D$,

- the $k$-PERFECT indePEndent DOMinAting SET problem, also known as the $k$-PERFECT CODE problem [22, 28, 47, 48], where the $k$-dominating set has to be perfect and independent, and

- the $k$-Total Perfect Dominating Set problem [47, 48], where the $k$ dominating set has to be total and perfect.

For all these instances, the condition of the following Theorem 29 holds and, hence, for these problems, we have an $O\left(c^{\sqrt{k}} n\right)$ time algorithm for some constant $c$. More precisely, a variant of Theorem 11 can be stated for each of these problems, leading to algorithms where the base $q$ of the exponential term is as follows:

- INDEPENDENT DOMINATING SET: $q=3$,

- total Dominating SET: $q=4$, since one must also distinguish for the vertices in the domination set whether or not they have been dominated by other vertices from the dominating set, 
- PERFECT DOMINATING SET: $q=3$; here, in contrast to the algorithm given in the proof of Theorem 11, a vertex is colored "grey" if it is dominated by exactly one "black" vertex which either lies in the "current" bag of the tree decomposition algorithm or in one of its child bags,

- PERFECT CODE: $q=3$, and

- TOtal PERFECT DOMinATing SET: $q=4$.

More details on the structure of the tables within the algorithm using the tree decomposition can be found in $[47,48]$. Observe that the table combination technique exhibited in Theorem 11 yields a basis in the exponential term of the running time which is only the square root of the basis obtained by Telle and Proskurowski in $[47,48]$ for these variants.

Note that all the problems listed above are defined via properties $P$ which can be viewed as being "local properties." This means that essential information for deciding $P$ can be maintained by the algorithms based on the tree decomposition approach. On the other hand, the CONNECTED DOMINATING SET problem (where it is required that the dominating set which is looked for should be connected) does not seem to be solvable in this way, since connectedness cannot be decided on the basis of knowing only a subset of the dominating set.

All the problem variants listed above are known to be NP-complete problems $[47,48]$ for general graphs. As regards their parameterized complexity, it is only known that $k$-PERFECT CODE (on general graphs) is a W[2]-problem which is W[1]-hard, and $k$-INDEPENDENT DOMINATING SET (on general graphs) is W[2]complete, see [22]. The other variants are not mentioned in [22]. In spite of these hardness results, we can obtain algorithms which have a running time of the form $O\left(c^{\sqrt{k}} n\right)$ for some constant $c$ for all of the instances of $k$-DOMINATING SET WITH PROPERTY $P$ when restricting oneself to planar graphs. More generally, we can state:

Theorem 29 Suppose that there is an algorithm that solves in $O\left(q^{\ell} N\right)$ time the DOMINATING SET WITH PROPERTY $P$ problem on graphs, given a tree decomposition with treewidth $\ell$ and $N$ nodes for some constant $q$. Then, the $k$-DOMINATING SET WITH PROPERTY $P$ problem can be solved in $O\left(q^{d \sqrt{k}} n\right)$ time, given a planar graph $G=(V, E)$, where $d=6 \sqrt{34}$ and $n=|V|$. Moreover, if a $k$-dominating set with property $P$ exists, a minimum size dominating set with property $P$ can be constructed within the same time.

Proof. If the planar graph $G$ admits a dominating set with property $P$ of size at most $k$, then, clearly, $G$ has domination number at most $k$. By Theorem 27, the treewidth of $G$ is bounded by $6 \sqrt{34} \sqrt{k}+8$. According to the discussion in Section 4 , a corresponding tree decomposition with $O(n)$ nodes can be found in time $O(\sqrt{k} n)$. The assumption on the existence of an $O\left(q^{\ell} N\right)$ time algorithm for given tree decomposition of width $\ell$ then yields the claim. 


\subsection{Weighted DOMinating SET}

Here, we consider the following variant of DOMINATING SET: Take a graph $G=$ $(V, E)$ together with an integer weight function $w: V \rightarrow \mathbb{N}$ with $w(v)>0$ for all $v \in V$. The weight of a vertex set $D \subseteq V$ is defined as $w(D)=\sum_{v \in D} w(v)$. A $k$-weighted dominating set $D$ of an undirected graph $G$ is a dominating set $D$ of $G$ with $w(D) \leq k$. The $k$-WEIGHTED DOMINATING SET problem is the task to decide, given a graph $G=(V, E)$, a weight function $w: V \rightarrow \mathbb{N}$, and a positive integer $k$, whether or not there exists a $k$-weighted dominating set.

Theorem 30 The PLANAR $k$-WEIGHTED DOMINATING SET problem can be solved in time $O\left(c^{\sqrt{k}} n\right)$, where $n$ is the size of the graph, and $c=3^{6 \sqrt{34}}$. Moreover, if a k-weighted dominating set exists, a dominating set of minimum weight can be constructed within the same time.

Proof. By definition of the integer weight function, a graph which possesses a $k$-weighted dominating set has a domination number bounded by $k$. Hence, its treewidth is bounded by $6 \sqrt{34 k}+8$, see Theorem 27 . Now, the techniques explained in Theorem 28 based on Theorem 11 work accordingly. In particular, only small modifications in the bookkeeping technique used in Theorem 11 are necessary in order to obtain a $k$-weighted dominating set. More precisely, we have to adapt the initialization of the variables $\min _{j}^{(i)}$ according to:

$$
\min _{j}^{(i)} \leftarrow\left\{\begin{array}{l}
+\infty, \quad \text { if the coloring represented by row } j \text { is non-valid } \\
w\left(\left\{x_{t}^{(i)} \mid t \in\left\{1, \ldots, n_{i}\right\}, c_{j, t}^{(i)}=0\right\}\right), \quad \text { otherwise. }
\end{array}\right.
$$

The updating of the variables $\min _{j}^{(i)}$ is adapted similarly.

Also weighted variants of domination problems with property $P$ can be treated in this way. Observe that it is not straightforward to generalize the theorem above to domination problems with rational or "real" weights. The natural reduction to the integer weight problem treated above would also influence the parameter $k$, which then would depend on the whole problem instance, which is not feasible in the fixed parameter setting. Only if we fix beforehand a constant $\epsilon$ such that all admissible positive weights are greater than $\epsilon$, this reduction would be feasible, showing that of the corresponding generalized problem instance for PLANAR $k$ WEIGHTED DOMINATING SET is fixed parameter tractable.

\subsection{FACE COVER}

We now turn our attention to the FACE COVER problem (see $[7,22,45])$. A $k$ face cover $C$ of an undirected plane graph $G=(V, E)$ (i.e., a planar graph with a fixed embedding) is a set of faces that cover all vertices of $G$, i.e., for every vertex $v \in V$, there exists a face $f \in C$ so that $v$ lies on the boundary of $f$. The $k$-FACE COVER problem is the task to decide, given a plane graph $G=(V, E)$ 
and a positive integer $k$, whether or not there exists a $k$-face cover. The size of a face cover of minimum size of a plane graph $G$ is called the face cover number of $G$. The FACE COVER problem is the task to find a minimum size face cover for a given plane graph.

Downey and Fellows [22, p.38] derived an $O\left(12^{k} n\right)$ algorithm for $k$-FACE COVER, which they call FACE COVER NUMBER FOR PLANAR GRAPHS. Before, Bienstock and Monma [7] had derived an $O\left(c^{k} n\right)$ algorithm (with an unspecified but large constant $c$ ) for a generalization of FACE COVER which they call DISK DIMENSION problem.

Basically, Downey and Fellows reduce FACE COVER to a problem which they call PLANAR RED/BLUE DOMINATING SET. Actually, we will use the same reduction technique in the following. This justifies why we also briefly treat this auxiliary problem.

An instance of PLANAR RED/BLUE DOMINATING SET is given by a planar bipartite graph $G=(V, E)$, where the bipartition is given by $V=V_{\text {red }} \cup V_{\text {blue }}$. The parameter is $k$. The question is whether there exists a set $V^{\prime} \subseteq V_{\text {red }}$ with $\left|V^{\prime}\right| \leq k$ such that every vertex of $V_{b l u e}$ is adjacent to at least one vertex of $V^{\prime}$.

Observe that PLANAR RED/BLUE DOMINATING SET is not a variant of planar dominating set in the sense of the first subsection, because a solution $V^{\prime}$ is not a dominating set, since red vertices can not and hence need not be dominated by red vertices. Downey and Fellows [22, p.38] give an $O\left(12^{k} n\right)$ algorithm for PLANAR RED/BLUE DOMINATING SET.

Although it would surely be possible to develop an analogue to Theorem 28 for PLANAR RED/BLUE DOMINATING SET as well, we will only state and prove the following version here, since we are mainly interested in solving the FACE COVER problem.

Lemma 31 Let a (planar) bipartite graph $G=(V, E)$ with bipartition $V=$ $V_{\text {red }} \cup V_{\text {blue }}$ be given together with a tree decomposition of width $\ell$. Then RED/BLUE DOMINATING SET can be solved in time $O\left(2^{\ell} N\right)$, where $N$ is the number of bags of the tree decomposition.

Proof. Basically, the technique exhibited in Theorem 11 can be applied. Due to the bipartite nature of the graph, only two "states" have to be stored for each vertex: red vertices are either within the dominating set or not, and blue vertices are either already dominated or not yet dominated. Therefore, we can derive base 2 instead of 3 for the exponential term of the claimed running time. The apparent technical details are left to the reader.

In order to solve the FACE COVER problem with the previously established techniques, we need the following auxiliary notion. Let $G=(V, E)$ be a plane graph. Let $F$ denote the set of faces of $G$. A mapping $r: F \rightarrow V$ is called a c-bounded face representation iff for all $v \in r(F)$ :

1. for all $f \in r^{-1}(v), v$ lies on the boundary of $f$, and 
2. $\left|r^{-1}(v)\right| \leq c$.

Lemma 32 Each plane graph has a 5-bounded face representation. Moreover, such a face representation function can be constructed in time $O\left(n^{2}\right)$, where $n$ is the number of vertices of the given graph.

Proof. Let $G_{0}=\left(V_{0}, E_{0}\right)$ be a plane graph. We are going to define a 5 -bounded face representation $r$ of $G_{0}$ in a step-by-step fashion.

It is well-known that each planar graph has a vertex of degree of at most five [43, Corollary 1.4]. Hence, select one of these low-degree-vertices of $G_{0}$ and call it $v_{0}$. Since $v_{0}$ has degree at most five, there are at most five faces adjacent to $v_{0}$. For all these faces $f$, we define $r(f)=v_{0}$.

Consider, then, the graph $G_{1}=\left(V_{1}, E_{1}\right)$, where $G_{1}=G_{0}-v_{0}$. We assume the "same" planar embedding for $G_{1}$ as for $G_{0}$. As before, we can find a vertex $v_{1} \in V_{1}$ with at most five adjacent faces (in $G_{1}$ ). Therefore, $v_{1}$ has at most five adjacent faces in $G_{0}$ to which no vertices have yet been assigned. For all these faces $f$, we define $r(f)=v_{1}$.

Inductively, $G_{i+1}$ is obtained as $G_{i}-v_{i}$, where $v_{i}$ has degree of at most five in $G_{i}$. Here, $v_{i}$ represents all adjacent faces in $G_{0}$ which are not already represented by the previously selected vertices $v_{0}, \ldots, v_{i-1}$.

This loop is repeated until all faces $f$ of $G_{0}$ have one representing vertex $r(f)$, i.e., until $r$ is completely defined.

The claimed time bound is obvious.

The preceding lemma will be the key in the proof of the following theorem.

Theorem 33 The $k$-FACE COVER problem can be solved in time $O\left(c_{1}^{\sqrt{k}} n+n^{2}\right)$, where $n$ is the size of the given plane graph, and $c_{1}=2^{36 \sqrt{34}}$. Moreover, if the face cover number of the input graph is at most $k$, then a minimum size face cover can be constructed within the same time.

Proof. Let $G=(V, E)$ be a plane graph with face set $F$. Due to Lemma 32, we can find a 5-bounded face representation $r: F \rightarrow V$ in time $O\left(n^{2}\right)$.

Consider the following graph: Add a vertex to each face of $G$, and make each such "face vertex" adjacent to all vertices that are on the boundary of that face. These are the only edges of the bipartite graph $G^{\prime}=\left(V^{\prime}, E^{\prime}\right)$. Write $V^{\prime}=V \cup V_{F}$, where $V_{F}$ is the set of face vertices, i.e., each $v \in V_{F}$ represents a face $f_{v}$ in $G$. In other words, $V$ and $V_{F}$ form the bipartition of $G^{\prime}$. Observe that $G^{\prime}$ can be viewed as an instance of RED-BLUE DOMINATING SET: the face vertices $V_{F}$ are "red" and the other vertices $V$ are "blue." Basically, this is the idea Downey and Fellows indicated in [22, Exercise 3.1.5]. Now, we would like to apply Lemma 31 to finish the proof.

To this end, consider the graph $\hat{G}=(V, \hat{E})$ obtained from $G^{\prime}$ by contracting each edge connecting a face vertex $v \in V_{F}$ of $G^{\prime}$ with $r\left(f_{v}\right)$. In other words, 
$\hat{G}$ is obtained from $G$ by firstly removing all "original" edges from $E$, and then inserting, for every $f \in F$, edges between $r(f)$ and each other vertex $v$ adjacent to $f$ in $G$.

Now, assume that $G$ has a $k$-face cover. Then, $\hat{G}$ has a $k^{\prime}$-dominating set with $k^{\prime} \leq k$. Namely, if $C \subseteq F$ is a face cover of $G$, then $r(C)$ is a dominating set of $\hat{G}$. Therefore, Theorem 27 yields $\operatorname{tw}(\hat{G}) \in O(\sqrt{k})$. We consider one tree decomposition of $\hat{G}$ of width $\leq 6 \sqrt{34 k}$. In order to be able to apply Lemma 31 , we are going to construct a tree decomposition of $G^{\prime}$ of width $\leq 36 \sqrt{34 k}$ from the tree decomposition of $\hat{G}$. To this end, we enhance the bags of $\hat{G}$ 's tree decomposition according to the following rule: if $r(f)$ is in some bag for some face $f \in F$, then put all $v \in V_{F}$ into that bag which satisfies $r\left(f_{v}\right)=r(f)$. The reader should verify that this, indeed, yields a tree decomposition of $G^{\prime}$, and the claimed width bound follows from Lemma 32.

Hence, our algorithm for FACE COVER leads to an asymptotic improvement of the result of Downey and Fellows [22, p.38]. The DISK DIMENSION problem treated by Bienstock and Monma [7] generalizes FACE COVER in two ways: firstly, they do not start with a fixed embedding of the planar input graph and, secondly, they have an additional input of their problem, namely a set $D$ of designated vertices, where only these need to be covered. Both of these generalizations seem to be hard to treat within our framework.

\section{Conclusion}

In this paper, we presented a treewidth-based approach to improve the fixed parameter complexity of PLANAR $k$-DOMINATING SET, $k$-FACE COVER and related problems drastically - we gained a significant exponential improvement over previous exact solutions for the problems. Seemingly for the first time, our results provide fixed parameter algorithms whose exponential factor has an exponent sublinear in the parameter.

The results. We show that for the treewidth of planar graphs, we have $\operatorname{tw}(G) \in$ $O(\sqrt{\gamma(G)})$, where $\gamma(G)$ is the domination number of $G$. From this, we derive that Planar $k$-DOMinating SET can be solved in time $O\left(c^{\sqrt{k}} n\right)$, where $c=3^{6 \sqrt{34}}$. Analogously, PLANAR $k$-INDEPENDENT DOMINATING SET, PLANAR $k$-PERFECT DOMINATING SET, PLANAR $k$-PERFECT CODE, and PLANAR $k$-WEIGHTED DOMINATING SET can be solved in the same time bounds and PLANAR $k$-TOTAL DOMINATING SET and PLANAR $k$-TOTAL PERFECT DOMINATING SET can be

solved in time $O\left(c_{1}^{\sqrt{k}} n\right)$, where $c_{1}=4^{6 \sqrt{34}}$. Finally, the $k$-FACE COVER problem can be solved in time $O\left(c_{2}^{\sqrt{k_{1}}} n+n^{2}\right)$, where $c_{2}=2^{36 \sqrt{34}}$. 
The constants. As already discussed earlier in the paper, our proven constants are huge. Hence, our result might be of only structural importance. There is hope for getting our results to be more practical, however: Our analysis only deals with worst case bounds. Even these might be improved significantly. A matter of special importance in this context is to note that our bounds heavily depend on the layer decomposition of the given planar graph, which seems to be a good starting point for the fight for better constants. In summary, ameliorating the constants needs and deserves future work.

The philosophical matters. As our results suffer from bad constants, besides discussing the opportunities to improve these, it is also valuable to investigate the "philosophical" perspectives opened by our work. Does our result mean that PLANAR $k$-DOMINATING SET is an "easy" fixed parameter tractable problem, e.g., easier than (the classical parameterized problem) $k$-VERTEX COVER on general graphs? In theory, seemingly yes, because the asymptotics of the known exponential term for PLANAR $k$-DOMINATING SET is much better than for $k$ VERTEX COVER ("sublinear versus linear"); in practice, seemingly no, because for probably all reasonable sizes of $k$ (say, e.g., $k \approx 100$ ) the exponential term for $k$-VERTEX COVER (currently already smaller than $1.3^{k}[14,41]$ ) is much better. Does this imply something for the theory of fixed parameter tractability [22] as a whole? A different matter is "how sublinear" we can get. Is there an NP-hard, natural parameterized problem that allows exponents of $o(\sqrt{k})$ ? Or is there a nontrivial lower bound, maybe in relation with classical complexity theory (cf., e.g., known bounds for the 3-SATISFIABILITY problem)? Also, is there a general relation to the approximability properties of the corresponding problems?

The concrete open questions. It would be interesting to investigate experimentally the practical usefulness of our result, since our estimates for the constants are worst case and very pessimistic ones. It also would be interesting to see if these results can be extended to more variants of Dominating SeT and to other graph classes (e.g., graphs of bounded genus ${ }^{3}$ ). Another interesting open problem is how to use the techniques of this paper for the variant of the FACE COVER problem, where the embedding is not given as a input (i.e., for a given planar graph, find an embedding with minimum number of faces that cover all the vertices, cf. [7]). Finally, as a matter of practical importance it would also be interesting to get a time $O\left(c^{k} n\right)$ (search tree) algorithm for PLANAR $k$-DOMINATING SET with constant $c$ significantly smaller than 11 , the constant obtained by the search tree algorithm of Downey and Fellows [21, 22]. Moreover, the question whether PLANAR $k$-DOMINATING SET admits a problem kernel of small size still remains unanswered. Both issues, finding a better search tree algorithm and a

\footnotetext{
${ }^{3}$ The logical framework of Frick and Grohe [26] already guarantees fixed parameter tractability for this problem.
} 
small problem kernel, could be of high practical relevance, in particular in combination with the interleaving techniques presented in [42].

Acknowledgements. We are indebted to Ton Kloks for starting this research with us and for many discussions on the topic of this paper. We thank Peter Rossmanith for discussions on different approaches to solving PLANAR DOMINATING SET. This paper also profited from the comments of anonymous referees of the conference SWAT 2000.

\section{References}

[1] J. Alber, J. Gramm, and R. Niedermeier. Faster exact solutions for hard problems: a parameterized point of view. Accepted for Discr. Math., August 2000 .

[2] R. B. Allan and R. Laskar. On domination and independent domination numbers of a graph. Discr. Math. 23:73-76, 1978.

[3] B. Aspvall, A. Proskurowski, and J. A. Telle. Memory requirements for table computations in partial $k$-tree algorithms. Algorithmica 27: 382-394, 2000.

[4] G. Ausiello, P. Crescenzi, G. Gambosi, V. Kann, A. Marchetti-Spaccamela, and M. Protasi. Complexity and Approximation. Springer-Verlag, 1999.

[5] B. S. Baker. Approximation algorithms for NP-complete problems on planar graphs. Journal of the ACM 41:153-180, 1994.

[6] A. E. Barkausas. Efficient dominating sets in spanning trees. J. Comb. Math. Comb. Comput. 15: 129-140, 1994.

[7] D. Bienstock and C. L. Monma. On the complexity of covering vertices by faces in a planar graph. SIAM J. Comput. 17(1):53-76, 1988.

[8] H. L. Bodlaender. A linear time algorithm for finding tree-decompositions of small treewidth. SIAM J. Comput. 25:1305-1317, 1996.

[9] H. L. Bodlaender. Treewidth: Algorithmic techniques and results. In Proceedings 22nd MFCS'97\%, Springer-Verlag LNCS 1295, pp. 19-36, 1997.

[10] H. L. Bodlaender. A partial $k$-arboretum of graphs with bounded treewidth. Theor. Comp. Sci. 209:1-45, 1998.

[11] A. Brandstädt, V. B. Le, and J. P. Spinrad. Graph Classes: a Survey. SIAM Monographs on Discrete Mathematics and Applications. Society for Industrial and Applied Mathematics, Philadelphia, 1999. 
[12] H. J. Broersma, T. Kloks, D. Kratsch, and H. Müller. Independent sets in AT-free graphs. In Proceedings 24th ICALP'97, Springer-Verlag LNCS 1256, pp. 760-770, 1997.

[13] M. S. Chang. Efficient algorithms for the domination problem on interval and circular arc graphs. SIAM J. Comput. 27:1671-1694, 1998.

[14] J. Chen, I. Kanj, and W. Jia. Vertex cover: further observations and further improvements. In Proceedings 25th WG, Springer-Verlag LNCS 1665, pp. 313-324, 1999.

[15] D. G. Corneil and L. K. Stewart. Dominating sets in perfect graphs. Discr. Math. 86:145-164, 1990.

[16] P. Crescenzi and V. Kann. A compendium of NP optimization problems. Available at http://www.nada.kth.se/theory/problemlist.html, August 1998 .

[17] M. Damian-Iordache and S.V. Pemmaraju. A $(2+\epsilon)$-approximation scheme for minimum domination on circle graphs. In Proceedings 11th ACM-SIAM Symposium on Discrete Algorithms 2000, pp. 672-679, 2000.

[18] G. Di Battista, P. Eades, R. Tamassia, and I.G. Tollis, Graph Drawing; Algorithms for the Visualization of Graphs, Prentice Hall, Upper Saddle River, NJ, USA, 1999.

[19] R. Diestel. Graph Theory, Graduate Texts in Mathematics 173, SpringerVerlag, 1997.

[20] R. G. Downey and M. R. Fellows. Fixed-parameter tractability and completeness. Congr. Num. 87:161-187, 1992.

[21] R. G. Downey and M. R. Fellows. Parameterized computational feasibility. In P. Clote, J. Remmel (eds.): Feasible Mathematics II, pp. 219-244. Birkhäuser, 1995.

[22] R. G. Downey and M. R. Fellows. Parameterized Complexity. SpringerVerlag, 1999.

[23] R. G. Downey and M. R. Fellows. Parameterized complexity after (almost) ten years: Review and open questions. In Combinatorics, Computation \& Logic, DMTCS'99 and CATS'99, Australian Computer Science Communcations, Volume 21 Number 3, pages 1-33. Springer-Verlag Singapore, 1999.

[24] R. G. Downey, M. R. Fellows, and U. Stege. Parameterized complexity: A framework for systematically confronting computational intractability. DIMACS Series in Discrete Mathematics and Theoretical Computer Science, 49:49-99, 1999. 
[25] R. D. Dutton and R. C. Brigham. Domination in claw-free graphs. Congr. Num. 132: 69-75, 1998.

[26] M. Frick and M. Grohe. Deciding first-order properties of locally treedecomposable structures. In Proceedings 26th ICALP'99, Springer-Verlag LNCS 1644, pp. 331-340, 1999.

[27] M. Garey and D. Johnson. Computers and Intractability: A Guide to the Theory of NP-completeness. Freeman, 1979.

[28] M. M. Halldórsson, J. Kratochvíl, and J. A. Telle. Independent sets with domination constraints. In Proceedings 25th ICALP'98, Springer-Verlag LNCS 1443, pp. 176-187, 1998.

[29] T. W. Haynes. Domination in graphs: a brief overview. J. Comb. Math. Comb. Comput. 24:225-237, 1997.

[30] T. W. Haynes, S. T. Hedetniemi, and P. J. Slater (eds.), Domination in graphs. Monographs and textbooks in pure and applied Mathematics, Marcel Dekker, 209, New York, 1998.

[31] M. A. Henning. Domination in graphs, a survey. Congr. Num. 116:139-179, 1996.

[32] D. S. Hochbaum (ed.). Approximation algorithms for NP-hard problems. Boston, MA: PWS Publishing Company, 1997.

[33] A. Kanevsky. Finding all minimum-size separating vertex sets in a graph. Networks 23: 533-541, 1993.

[34] M. Keil. The complexity of domination problems in circle graphs. Discr. Appl. Math. 42:51-63, 1993.

[35] D. Kratsch and L. Stewart. Domination of cocomparability graphs. SIAM J. Discrete Math. 6:400-417, 1993.

[36] J. van Leeuwen. Graph Algorithms. In Handbook of Theoretical Computer Science, Vol. A: Algorithms and Complexity, pp. 525-631, North Holland, 1990 .

[37] G. MacGillivray and K. Seyffarth. Domination numbers of planar graphs. J. Graph Theory 22:213-229, 1996.

[38] K. Mehlhorn. Graph Algorithms and NP-Completeness. Springer-Verlag, 1984.

[39] Z. Michalewicz and D. B. Fogel. How to solve it: Modern Heuristics. Springer-Verlag, 2000. 
[40] H. Müller and A. Brandstädt. The NP-completeness of Steiner TREE and DOMinating SET for chordal bipartite graphs. Theor. Comp. Sci. 53:257$265,1987$.

[41] R. Niedermeier and P. Rossmanith. Upper bounds for Vertex Cover further improved. In Proceedings 16th STACS'99, Springer-Verlag LNCS 1563, pp. 561-570, 1999.

[42] R. Niedermeier and P. Rossmanith. A general method to speed up fixedparameter algorithms. Information Processing Letters 73:125-129, 2000.

[43] T. Nishizeki and N. Chiba. Planar graphs: theory and applications. Annals of Discr. Math., 32, North-Holland, 1988.

[44] C. H. Papadimitriou. Computational Complexity. Addison-Wesley, 1994.

[45] R. C. Read. Prospects for graph theory algorithms. Ann. Discr. Math. 55:201-210, 1993.

[46] J. A. Telle. Complexity of domination-type problems in graphs. Nordic J. Comput. 1:157-171, 1994.

[47] J. A. Telle and A. Proskurowski. Practical algorithms on partial $k$-trees with an application to domination-like problems. In Proceedings 3rd WADS'93, Springer-Verlag LNCS 709, pp. 610-621, 1993.

[48] J. A. Telle and A. Proskurowski. Algorithms for vertex partitioning problems on partial $k$-trees. SIAM J. Discr. Math. 10(4):529-550, 1997.

[49] K. Tohru, N. Yoshida, and Y. Kakuda. A linear time algorithm for the domination number of a series parallel graph. Discr. Appl. Math. 5:299-311, 1993. 